\title{
Sub-leading asymptotics of ECH capacities
}

\author{
Dan Cristofaro-Gardiner ${ }^{1} \cdot$ Nikhil Savale $^{2}$
}

Accepted: 30 August 2020 / Published online: 24 September 2020

(c) The Author(s) 2020

\begin{abstract}
In previous work (Cristofaro-Gardiner et al. in Invent Math 199:187-214, 2015), the first author and collaborators showed that the leading asymptotics of the embedded contact homology spectrum recovers the contact volume. Our main theorem here is a new bound on the sub-leading asymptotics.
\end{abstract}

Mathematics Subject Classification 53D35 · 57R57 · 57R58

\section{Introduction}

Asymptotic counting problems for length spectra are of significant interest in geometry, dynamics and number theory. As an example, let $\pi(x)$ denote the counting function for the geodesic length spectrum, with lengths $\leq \ln x$, on a Riemannian manifold of negative sectional curvature. Then a classical result of Margulis [29] states

$$
E(x):=\pi(x)-\operatorname{li}\left(x^{h}\right)=o\left(\operatorname{li}\left(x^{h}\right)\right)
$$

as $x \rightarrow \infty$, with $\operatorname{li}(y):=\int_{2}^{y} \frac{d s}{\ln s} \sim \frac{y}{\ln y}$ and $h$ being the topological entropy of the corresponding geodesic flow. Recently, [20, Theorem 2.8] improved the error estimate above, under a pinching condition, to $O\left(\operatorname{li}\left(x^{h-\alpha}\right)\right)$ for some $\alpha>0$ with the sharp exponent being yet unknown. In the case of $P S L_{2}(\mathbb{Z}) \backslash \mathbb{H}$, a surface of constant negative curvature -1 where $h=1$, there is a long history of improvements

D.C.-G. is partially supported by NSF Grant 1711976 . N.S. is partially supported by the DFG funded Project CRC/TRR 191.

$\bowtie \quad$ Nikhil Savale

nsavale@math.uni-koeln.de

Dan Cristofaro-Gardiner

dcristof@ucsc.edu

1 Department of Mathematics, University of California Santa Cruz, Santa Cruz, CA 95064, USA

2 Mathematisches Institut, Universität zu Köln, Weyertal 86-90, 50931 Cologne, Germany

Birkhäuser 
for the error term: $E(x)=O\left(x^{\frac{35}{48}+\varepsilon}\right)$ [24], $E(x)=O\left(x^{\frac{7}{10}+\varepsilon}\right)$ [28, Theorem 1.4], $E(x)=O\left(x^{\frac{71}{102}+\varepsilon}\right)$ [9] and $E(x)=O\left(x^{\frac{25}{36}+\varepsilon}\right)$ [38] with the conjectured $E(x)=$ $O\left(x^{\frac{1}{2}+\varepsilon}\right)$ expected because the Riemann hypothesis for a corresponding dynamical zeta function holds. Furthermore, counting results under homological constraints have also been proved in $[1,25,27,31,32]$.

The aim of the present paper is to explore an analogous problem in contact dynamics corresponding to asymptotic counting of the ECH spectrum. More precisely, let $Y$ be an oriented three-manifold. A contact form on $Y$ is a one-form $\lambda$ satisfying

$$
\lambda \wedge d \lambda>0
$$

A contact form determines the Reeb vector field, $R$, defined by

$$
\lambda(R)=1, \quad d \lambda(R, \cdot)=0,
$$

and the contact structure $\xi:=\operatorname{Ker}(\lambda)$. Closed orbits of $R$ are called Reeb orbits. For unit cosphere bundles over Riemannian surfaces, for example, there is a canonical contact form such that Reeb orbits correspond with closed geodesics on the surface. At present, it is not even known when precisely there are infinitely many simple closed Reeb orbits on a given contact manifold $[14,15]$.

If $Y$ is closed and $(Y, \lambda)$ is further equipped with a homology class $\Gamma \in H_{1}(Y)$, then the embedded contact homology $\operatorname{ECH}(Y, \lambda, \Gamma)$ is defined. This is the homology of a chain complex freely generated over $\mathbb{Z}_{2}$ by certain sets of Reeb orbits in the homology class $\Gamma$, relative to a differential that counts certain $J$-holomorphic curves in $\mathbb{R} \times Y$. (ECH can also be defined over $\mathbb{Z}$, but for the applications in this paper we will not need this.) It is known that the homology only depends on $\xi$, and so we sometimes denote it $\operatorname{ECH}(Y, \lambda, \xi)$. Any Reeb orbit $\gamma$ has a period/symplectic action

$$
\mathcal{A}(\gamma)=\int_{\gamma} \lambda
$$

and this induces a filtration on $E C H(Y, \lambda)$; we can use this filtration to define a number $c_{\sigma}(Y, \lambda)$ for every nonzero class in $\mathrm{ECH}$, called the spectral invariant associated to $\sigma$; the spectral invariants are $C^{0}$ continuous and so can be extended to degenerate contact forms as well. We will review the definition of $\mathrm{ECH}$ and of the spectral invariants in Sect. 2.1.

When the class $c_{1}(\xi)+2$ P.D. $(\Gamma) \in H^{2}(Y ; \mathbb{Z})$ is torsion, then $E C H(Y, \xi, \Gamma)$ has a relative $\mathbb{Z}$ grading, which we can refine to a canonical absolute grading gr $\mathbb{Q}^{\mathbb{Q}}$ by rationals [26], and which we will review in Sect. 2.3. It is known that for large gradings the group is eventually 2-periodic and non-vanishing:

$$
E C H_{*}(Y, \xi, \Gamma)=E C H_{*+2}(Y, \xi, \Gamma) \neq 0, * \gg 0 .
$$


The main theorem of [16] states that in this case, the asymptotics of the spectral invariants recover the contact volume

$$
\operatorname{vol}(Y, \lambda)=\int_{Y} \lambda \wedge d \lambda .
$$

Specifically:

Theorem 1 [16, Theorem 1.3] Let $(Y, \lambda)$ be a closed, connected oriented threemanifold with a contact form, and let $\Gamma \in H_{1}(Y)$ be such that $c_{1}(\xi)+2 P . D .(\Gamma)$ is torsion. For any sequence $\left\{\sigma_{j}\right\}_{j=1}^{\infty}$ of nonzero classes in $\operatorname{ECH}(Y, \xi, \Gamma)$ with gradings $\operatorname{gr}^{\mathbb{Q}}\left(\sigma_{j}\right)=q+2 j, q \in \mathbb{Q}$, one has

$$
\lim _{j \rightarrow \infty} \frac{c_{\sigma_{j}}(Y, \lambda)^{2}}{2 j}=\operatorname{vol}(Y, \lambda) .
$$

The formula (1.2) has had various implications for dynamics. For example, it was a crucial ingredient in recent work [15] of the first author and collaborators showing that many Reeb vector fields on closed three-manifolds have either two or infinitely many distinct closed orbits, and it was used in [14] to show that every Reeb vector field on a closed three-manifold has at least two distinct closed orbits. It has also been used to prove $C^{\infty}$ closing lemmas for Reeb flows on closed three-manifolds and Hamiltonian flows on closed surfaces [2,23].

The Eq. (1.2) can be rewritten

$$
e(j):=[2 \operatorname{vol}(Y, \lambda)]^{1 / 2} j^{1 / 2}-c_{\sigma_{j}}(Y, \lambda)=o\left(j^{1 / 2}\right),
$$

as $j \rightarrow \infty$. It is then natural to ask for better remainder/error estimates on $e(j)$. To this end we shall prove.

Theorem 2 Let $(Y, \lambda)$ be a closed, connected oriented three-manifold with contact form $\lambda$, and let $\Gamma \in H_{1}(Y)$ be such that $c_{1}(\xi)+2 P . D$. $(\Gamma)$ is torsion. For any sequence $\left\{\sigma_{j}\right\}_{j=1}^{\infty}$ of nonzero classes in $\operatorname{ECH}(Y, \xi, \Gamma)$ with gradings $g r^{\mathbb{Q}}\left(\sigma_{j}\right)=q+2 j, q \in \mathbb{Q}$, one has the asymptotic bound $e(j)=O\left(j^{2 / 5}\right)$ on (1.3) as $j \rightarrow \infty$.

Previously, W. Sun has shown $e(j)=O\left(j^{125 / 252}\right)$ [43, Theorem 2.8].

We do not know whether or not the $O\left(j^{2 / 5}\right)$ asymptotics here are optimal-in other words, we do not know whether there is some contact form on a three-manifold realizing these asymptotics. We will show in Sect. 4.3 that there exist contact forms with $O(1)$ asymptotics for the $e(j)$. In Remark 12, we clarify where the exponent $\frac{2}{5}$ comes from in our proof, and why the methods in the current paper can not improve on it.

It is natural to ask whether the asymptotics of the $e(j)$ carry interesting geometric information. In this regard, a similar question in the context of the spectral flow of a one-parameter family of Dirac operators was recently answered in [37]. This is particularly relevant in the context of the argument we give here, as our argument also 
involves estimating spectral flow, see Remark 12 . Here we compute the asymptotics of the $e(j)$ in a specific example, see Sect. 4.3, and connect the answer to the theory of symplectic embedding problems in Sect. 4.4; further computations and potential applications to embedding problems are planned for future work. In connection with dynamics, Sun [43] has also asked whether a quantitative refinement of the closing lemma is possible, which we also plan to explore.

\subsection{Weyl law and dynamical zeta function}

Our main Theorem 2 has two corollaries to the counting function for ECH spectrum and a corresponding zeta function which we now mention.

Given $\Gamma \in H_{1}(Y)$, define a set of nonnegative real numbers, called the $E C H$ spectrum for $(Y, \lambda, \Gamma)$

$$
\begin{aligned}
\Sigma_{(Y, \lambda, \Gamma)} & :=\cup_{*} \Sigma_{(Y, \lambda, \Gamma), *} \\
\Sigma_{(Y, \lambda, \Gamma), *} & :=\left\{c_{\sigma}(\lambda) \mid 0 \neq \sigma \in E C H_{*}\left(Y, \xi, \Gamma ; \mathbb{Z}_{2}\right)\right\} .
\end{aligned}
$$

(To emphasize, in the set $\Sigma_{(Y, \lambda, \Gamma), *}$ we are fixing the grading $*$.) Then, define the Weyl counting function for $\Sigma_{(Y, \lambda, \Gamma)}$

$$
N_{(Y, \lambda, \Gamma)}(R):=\#\left\{c \in \Sigma_{(Y, \lambda, \Gamma)} \mid c \leq R\right\} .
$$

We now have the following:

Corollary 3 If $c_{1}(\xi)+2 P . D .(\Gamma)$ is torsion, then the Weyl counting function (1.4) for the ECH spectrum satisfies the asymptotics

$$
N(R)=\left[\frac{2^{d}-1}{\operatorname{vol}(Y, \lambda)}\right] R^{2}+O\left(R^{9 / 5}\right)
$$

where

$$
d:=\operatorname{dim} E C H_{*}\left(Y, \xi, \Gamma ; \mathbb{Z}_{2}\right)+\operatorname{dim} E C H_{*+1}\left(Y, \xi, \Gamma ; \mathbb{Z}_{2}\right), * \gg 0 .
$$

As another corollary, one may obtain information on the corresponding dynamical zeta function. To this end, first note that the ECH zeta function

$$
\zeta_{E C H}(s ; Y, \lambda, \Gamma):=\sum_{0 \neq c \in \Sigma_{(Y, \lambda, \Gamma)}} c^{-s}
$$

converges for $\operatorname{Re}(s)>2$ by (1.2) and defines a holomorphic function of $s$ in this region whenever $c_{1}(\xi)+2$ P.D. $(\Gamma)$ is torsion, by (1.5).

In view of for example $[19,20]$, one can ask if $\zeta_{E C H}$ has a meromorphic continuation to $\mathbb{C}$, and, if so, whether it contains interesting geometric information. The Weyl law (1.5) then shows: 
Corollary 4 The zeta function (1.6) continues meromorphically to the region $\operatorname{Re}(s)>$ $\frac{5}{3}$. The only pole in this region is at $s=2$ which is further simple with residue $\operatorname{Res}_{S=2} \zeta_{E C H}(s ; Y, \lambda, \Gamma)=\left[\frac{2^{d}-1}{\operatorname{vol}(Y, \lambda)}\right]$.

In Sect. 4.3, we give an example of a contact form for which $\zeta_{E C H}$ has a meromorphic extension to all of $\mathbb{C}$ with two poles at $s=1,2$. The meromorphy and location of the poles of (1.6) would be interesting to figure out in general.

\subsection{Idea of the proof and comparison with previous works}

The method of the proof uses previous work by C. Taubes relating embedded contact homology to Monopole Floer homology. By using Taubes's results, we can estimate spectral invariants associated to nonzero ECH classes by estimating the energy of certain solutions of the deformed three-dimensional Seiberg-Witten equations. This is also the basic idea behind the proofs of Theorem 1 and the result of Sun mentioned above, and it was inspired by a similar idea in Taubes's proof of the three-dimensional Weinstein conjecture [40].

The essential place where our proof differs from these arguments involves a particular estimate, namely a key "spectral flow" bound for families of Dirac operators that appears in all of these proofs. This estimate bounds the difference between the grading of a Seiberg-Witten solution, and the "Chern-Simons" functional, which we review in Sect. 2.2, and is important in all of the works mentioned above. We prove a stronger bound of this kind than any previous bound, see Proposition 5 and the discussion about the eta invariant below, and this is the key point which allows us to prove $O\left(j^{2 / 5}\right)$ asymptotics. Spectral flow bounds for families of Dirac operators were also considered in [34-36,42]. The main difference here is that in those works the bounds were proved on reducible solutions where the connections needed to define the relevant Dirac operators were explicitly given. Here we must consider irreducible solutions, and so we rely on a priori estimates.

We have chosen to phrase this spectral flow bound in terms of a bound on the eta invariants of a family of operators. By the Atiyah-Patodi-Singer index theorem, the bound we need on the spectral flow is equivalent to a bound on the eta invariant, and we make the relationship between these two quantities precise in the appendix.

The paper is organized as follows. In Sect. 2, we review what we need to know about embedded contact homology, Monopole Floer cohomology and Taubes's isomorphism. Section 3 reviews the eta invariant, reviews the necessary estimates on irreducible solutions to the Seiberg-Witten equations, and proves the key Proposition 5. We then give the proof of Theorem 2 in Sect. 4-while our argument in this section is novel, one could instead argue here as in [43], but we give our own argument here since it might be of independent interest, see Remark 14. The end of the paper reviews the sub-leading asymptotics and the dynamical zeta function in the case of ellipsoids, and an appendix rephrases the grading in Seiberg-Witten in terms of the eta invariant rather than in terms of spectral flow. 


\section{Floer homologies}

We begin by reviewing the facts that we will need about ECH and Monopole Floer homology.

\subsection{Embedded contact homology}

We first summarize what we will need to know about ECH. For more details and for definitions of the terms that we have not defined here, see [22].

Let $(Y, \lambda)$ be a closed oriented three-manifold with a nondegenerate contact form. Fix a homology class $\Gamma \in H_{1}(Y)$. As stated in the introduction, the embedded contact homology $E C H(Y, \lambda, \Gamma)$ is the homology of a chain complex $E C C(Y, \lambda, \Gamma)$. To elaborate, the chain complex ECC is freely generated over $\mathbb{Z}_{2}$ by orbit sets $\alpha=$ $\left\{\left(\alpha_{j}, m_{j}\right)\right\}$ where the $\alpha_{j}$ 's are distinct embedded Reeb orbits while each $m_{j} \in \mathbb{N}$; we further have the constraints that $\sum m_{j} \alpha_{j}=\Gamma \in H_{1}(Y)$ and $m_{j}=1$ if $\alpha_{j}$ is hyperbolic. To define the chain complex differential $\partial$, we consider the symplectization $\left(\mathbb{R}_{t} \times Y, d\left(e^{t} \lambda\right)\right)$, and choose an almost complex structure $J$ that is $\mathbb{R}$-invariant, rotates the contact hyperplane $\xi:=\operatorname{ker} \lambda$ positively with respect to $d \lambda$, and satisfies $J \partial_{t}=R$. The differential on $\operatorname{ECC}(Y, \lambda, \Gamma)$ is now defined via

$$
\partial \alpha=\sum_{\beta} \underbrace{\sharp\left[\mathcal{M}_{1}(\alpha, \beta) / \mathbb{R}\right]}_{=:\langle\partial \alpha, \beta\rangle} \beta .
$$

Here $\mathcal{M}_{1}(\alpha, \beta)$ denotes the moduli space of $J$-holomorphic curves $C$ of ECH index $I(C)=1$ in the symplectization, modulo translation in the $\mathbb{R}$-direction, and modulo equivalence as currents, with the set of positive ends given by $\alpha$ and the set of negative ends given by $\beta$. If $J$ is generic, then the differential squares to zero $\partial^{2}=0$ and defines the ECH group $E C H(Y, \lambda ; \Gamma)$. We will not review the definition of the ECH index here, see [22] for more details, but the key point is that the condition $I(C)=1$ forces $C$ to be (mostly) embedded and rigid modulo translation.

As stated in the introduction, the homology $E C H(Y, \lambda ; \Gamma)$ does not depend on the choice of generic $J$, and only depends on the associated contact structure $\xi$; we therefore denote it $E C H(Y, \xi ; \Gamma)$. (In fact, the homology only depends on the $\operatorname{spin}^{c}$ structure determined by $\xi$, but we will not need that.) This follows from a canonical isomorphism between ECH and Monopole Floer homology [41], which we will soon review. The ECH index $I$ induces a relative $\mathbb{Z} / d \mathbb{Z}$ grading on $E C H(Y, \xi ; \Gamma)$, where $d$ is the divisibility of $c_{1}(\xi)+2$ P.D. $(\Gamma) \in H^{2}(Y ; \mathbb{Z})$ mod torsion. In particular, it is relatively $\mathbb{Z}$-graded when this second homology class is torsion

Recall now the action of a Reeb orbit from the introduction. This induces an action on orbit sets $\alpha=\left\{\left(\alpha_{j}, m_{j}\right)\right\}$ by

$$
\mathcal{A}(\alpha):=\sum_{j=1}^{N} m_{j}\left(\int_{\alpha_{j}} \lambda\right) .
$$


The differential decreases action, and so we can define $\operatorname{ECC}^{L}(Y, \lambda, \Gamma)$ to be the homology of the sub-complex generated by orbit sets of action strictly less than $L$. The homology of this sub-complex $E C H^{L}(Y, \lambda, \Gamma)$ is again independent of $J$ but now depends on $\lambda$; there is an inclusion induced map $E C H^{L}(Y, \lambda, \Gamma) \rightarrow E C H(Y, \xi, \Gamma)$. Using this filtration, we can define the spectral invariant associated to a nonzero class $\sigma$ in $\mathrm{ECH}$

$$
c_{\sigma}(Y, \lambda):=\inf \left\{L \mid \sigma \in \operatorname{image}\left(E C H^{L}(Y, \lambda, \Gamma) \rightarrow E C H(Y, \xi, \Gamma)\right)\right\} .
$$

As stated in the introduction, the spectral invariants are known to be $C^{0}$ continuous in the contact form, and so extend to degenerate contact forms as well by taking a limit over nondegenerate forms, see [21].

\subsection{Monopole Floer homology}

We now briefly review what we need to know about Monopole Floer homology, referring to [26] for additional details and definitions.

Recall that a $\operatorname{spin}^{c}$ structure on an oriented Riemannian three-manifold $Y$ is a pair $(S, c)$ consisting of a rank 2 complex Hermitian vector bundle and a Clifford multiplication endomorphism $c: T^{*} Y \otimes \mathbb{C} \rightarrow$ End $(S)$ satisfying $c\left(e_{1}\right)^{2}=-1$ and $c\left(e_{1}\right) c\left(e_{2}\right) c\left(e_{3}\right)=1$ for any oriented orthonormal frame $\left(e_{1}, e_{2}, e_{3}\right)$ of $T_{y} Y$. Let $\mathfrak{s u}(S)$ denote the bundle of traceless, skew-adjoint endomorphisms of $S$ with inner product $\frac{1}{2} \operatorname{tr}\left(A^{*} B\right)$. Clifford multiplication $c$ maps $T^{*} Y$ isometrically onto $\mathfrak{s u}(S)$. $\operatorname{Spin}^{c}$ structures exist on any three-manifold, and the set of $\operatorname{spin}^{c}$ structures is an affine space over $H^{2}(Y ; \mathbb{Z})$. A $\operatorname{spin}^{c}$ connection $A$ on $S$ is a connection such that $c$ is parallel. Given two spin-c connections $A_{1}, A_{2}$ on $S$, their difference is of the form $A_{1}-A_{2}=a \otimes 1^{S}$ for some $a \in \Omega^{1}(Y, i \mathbb{R})$. If we denote by $A_{1}^{t}, A_{2}^{t}$ the induced connections on $\operatorname{det}(S)=\Lambda^{2} S$, we then have $A_{1}^{t}-A_{2}^{t}=2 a$. Hence prescribing a $\operatorname{spin}^{c}$ connection on $S$ is the same as prescribing a unitary connection on $\operatorname{det}(S)$. We let $\mathcal{A}(Y, \mathfrak{s})$ denote the space of all $\operatorname{spin}^{c}$ connections on $S$. Given a $\operatorname{spin}^{c}$ connection $A$, we denote by $\nabla^{A}$ the associated covariant derivative. We then define the $\operatorname{spin}^{c}$ Dirac operator $D_{A}: C^{\infty}(S) \rightarrow C^{\infty}(S)$ via $D_{A} \Psi=c \circ \nabla^{A} \Psi$.

Given a $\operatorname{spin}^{c}$ structure $\mathfrak{s}=(S, c)$ on $Y$, monopole Floer homology assigns three groups denoted by $\widehat{H M}(Y, \mathfrak{s}), \widehat{H M}(Y, \mathfrak{s})$ and $\overline{H M}(Y, \mathfrak{s})$. These are defined via infinite dimensional Morse theory on the configuration space $\mathcal{C}(Y, \mathfrak{s})=\mathcal{A}(Y, \mathfrak{s}) \times$ $C^{\infty}(S)$ using the Chern-Simons-Dirac functional $\mathcal{L}$, defined as

$$
\mathcal{L}(A, \Psi)=\underbrace{-\frac{1}{8} \int_{Y}\left(A^{t}-A_{0}^{t}\right) \wedge\left(F_{A^{t}}+F_{A_{0}^{t}}\right)}_{=: C S(A)}+\frac{1}{2} \int_{Y}\left\langle D_{A} \Psi, \Psi\right\rangle d y
$$

using a fixed base spin-c connection $A_{0}$ (we pick one with $A_{0}^{t}$ flat in the case of torsion spin-c structures) and a metric $g^{T Y}$.

The gauge group $\mathcal{G}(Y)=\operatorname{Map}\left(Y, S^{1}\right)$ acts on the configuration space $\mathcal{C}(Y, \mathfrak{s})$ by $u .(A, \Psi)=\left(A-u^{-1} d u \otimes I, u \Psi\right)$. The gauge group action is free on the irre- 
ducible part $\mathcal{C}^{*}(Y, \mathfrak{s})=\{(A, \Psi) \in \mathcal{C}(Y, \mathfrak{s}) \mid \Psi \neq 0\} \subset \mathcal{C}(Y, \mathfrak{s})$ and not free along the reducibles. The blow up of the configuration space along the reducibles

$$
\mathcal{C}^{\sigma}(Y, \mathfrak{s})=\left\{(A, s, \Phi) \mid\|\Phi\|_{L^{2}}=1, s \geq 0\right\}
$$

then has a free $\mathcal{G}(Y)$ action $u \cdot(A, s, \Phi)=\left(A-u^{-1} d u \otimes I, s, u \Phi\right)$.

To define the Monopole Floer homology groups one needs to perturb the ChernSimons-Dirac functional (2.1). First given a one form $\mu \in \Omega^{1}(Y ; i \mathbb{R})$, one defines the functional $e_{\mu}(A):=\frac{1}{2} \int_{Y} \mu \wedge F_{A^{t}}$ whose gradient is calculated to be $* d \mu$. To achieve non-degeneracy and transversality of configurations one uses the perturbed Chern-Simons-Dirac functional

$$
\mathcal{L}_{\mu}(A, \Psi)=\mathcal{L}(A, \Psi)-e_{\mu}(A)
$$

where $\mu$ is a suitable finite linear combination of eigenvectors of $* d$ with non-zero eigenvalue. Next let

$$
\mathbb{T}=\left\{A \in \mathcal{A}(Y, \mathfrak{s}) \mid F_{A^{t}}=0\right\} / \mathcal{G}(Y)
$$

be the space of $A^{t}$ flat spin-c connections up to gauge equivalence. We choose a Morse function $f: \mathbb{T} \rightarrow \mathbb{R}$ to define the functional $\mathfrak{f}: \mathcal{C}^{\sigma}(Y, \mathfrak{s}) \rightarrow \mathbb{R}, \mathfrak{f}\left(A_{0}+a, s, \Psi\right):=$ $f\left(\left[A_{0}^{t}+a^{h}\right]\right)$, where $a^{h}$ denotes the harmonic part of $a \in \Omega^{1}(Y, i \mathbb{R})$. The gradient may be calculated $(\nabla \mathfrak{f})_{A}^{\sigma}=\left((\nabla f)_{\left.\left(A^{t}\right)^{h}, 0,0\right)}\right.$.

The Monopole Floer homology groups are now defined using solutions $(A, s, \Phi) \in$ $\mathcal{C}^{\sigma}(Y, \mathfrak{s})$ to the three-dimensional Seiberg-Witten equations

$$
\begin{aligned}
\frac{1}{2} * F_{A^{t}}+s^{2} c^{-1}\left(\Phi \Phi^{*}\right)_{0}+(\nabla f)_{p(A)}+* d \mu & =0 \\
s \Lambda(A, s, \Phi) & =0 \\
D_{A} \Phi-\Lambda(A, s, \Phi) \Phi & =0
\end{aligned}
$$

where $\Lambda(A, s, \Phi)=\left\langle D_{A} \Phi, \Phi\right\rangle_{L^{2}}$ and $\left(\Phi \Phi^{*}\right)_{0}:=\Phi \otimes \Phi^{*}-\frac{1}{2}|\Phi|^{2}$ defines a traceless, Hermitian endormophism of $S$. We denote by $\mathfrak{C}$ the set of solutions to the above equations.

We first subdivide the solutions as follows:

$$
\begin{aligned}
\mathfrak{C}^{o}= & \{(A, s, \Phi) \in \mathfrak{C} \mid s \neq 0\} / \mathcal{G}(Y), \\
\mathfrak{C}^{s}= & \{(A, 0, \Phi) \in \mathfrak{C} \mid \Lambda(A, 0, \Phi)>0\} / \mathcal{G}(Y) \\
= & \left\{(A, \Phi) \mid \frac{1}{2} F_{A^{t}}+d \mu=0,[A] \text { is a critical point of } f,\right. \\
& \left.\Phi \text { is a (positive-)normalized eigenvector of } D_{A}\right\} / \mathcal{G}(Y) \\
\mathfrak{C}^{u}= & \{(A, 0, \Phi) \mid \Lambda(A, 0, \Phi)<0\} / \mathcal{G}(Y) \\
= & \left\{(A, \Phi) \mid \frac{1}{2} F_{A^{t}}+d \mu=0,[A] \text { is a critical point of } f,\right.
\end{aligned}
$$


$\Phi$ is a (negative-)normalized eigenvector of $\left.D_{A}\right\} / \mathcal{G}(Y)$.

Next, we consider the free $\mathbb{Z}_{2}$ modules generated by the three sets above

$$
C^{o}=\mathbb{Z}_{2}\left[\mathfrak{C}^{o}\right], C^{s}=\mathbb{Z}_{2}\left[\mathfrak{C}^{s}\right], C^{u}=\mathbb{Z}_{2}\left[\mathfrak{C}^{u}\right]
$$

The chain groups for the three versions of Floer homology mentioned above are defined by

$$
\overleftarrow{C}=C^{o} \oplus C^{s}, \hat{C}=C^{o} \oplus C^{u}, \bar{C}=C^{s} \oplus C^{u}
$$

These chain groups $\widetilde{C}, \hat{C}, \bar{C}$ can be endowed with differentials $\check{\partial}, \hat{\partial}, \bar{\partial}$ with square zero; we do not give the precise details here, but the idea is to count Fredholm index one solutions of the four-dimensional equations, see [26, Theorem 22.1.4] for the details. The homologies of these three complexes are by definition the three monopole Floer homology groups

$$
\widehat{H M}(Y, \mathfrak{s}), \widehat{H M}(Y, \mathfrak{s}), \overline{H M}(Y, \mathfrak{s}) \text {. }
$$

They are independent of the choice of metric and perturbations $\mu, \mathfrak{f}$.

Each of the above Floer groups has a relative $\mathbb{Z} / d \mathbb{Z}$ grading where $d$ is the divisibility of $c_{1}(S) \in H^{2}(Y ; \mathbb{Z}) \bmod$ torsion. This is defined using the extended Hessian

$$
\begin{aligned}
\widehat{\mathcal{H}}_{(A, \Psi)}: C^{\infty}\left(Y ; i T^{*} Y \oplus \mathbb{R} \oplus S\right) & \rightarrow C^{\infty}\left(Y ; i T^{*} Y \oplus \mathbb{R} \oplus S\right) ;(A, \Psi) \in \mathcal{C}(Y, \mathfrak{s}) \\
\widehat{\mathcal{H}}_{(A, \Psi)}\left[\begin{array}{c}
a \\
f \\
\psi
\end{array}\right] & =\left[\begin{array}{c}
* d a+2 c^{-1}(\psi \Psi)_{0}-d f \\
-d^{*} a+i \operatorname{Re}\langle\psi, \Psi\rangle \\
c(a) \Psi+D_{A} \psi+f \Psi
\end{array}\right]\left[\begin{array}{c}
a \\
f \\
\psi
\end{array}\right] \\
& =\left[\begin{array}{ccc}
* d & -d & c^{-1}(. \Psi)_{0} \\
-d^{*} & 0 & \langle., \Psi\rangle \\
c(.) \Psi & . \Psi & D_{A}
\end{array}\right]\left[\begin{array}{l}
a \\
f \\
\psi
\end{array}\right] .
\end{aligned}
$$

The relative grading between two irreducible generators $\mathfrak{a}_{i}=\left(A_{i}, s_{i}, \Phi_{i}\right),\left(s_{i} \neq 0\right)$, $i=1,2$, is now defined via gr $\left(\mathfrak{a}_{1}, \mathfrak{a}_{2}\right)=\operatorname{sf}\left\{\widehat{\mathcal{H}}_{\left(A_{t}, \Psi_{t}\right)}\right\}_{0 \leq t \leq 1}(\bmod d)$ for some path of configurations $\left(A_{t}, \Psi_{t}\right)$ starting at $\left(A_{2}, s_{2} \Phi_{2}\right)$ and ending at $\left(A_{1}, s_{1} \Phi_{1}\right)$, where sf denotes the spectral flow.

In the case when the spin-c structure is torsion, the monopole Floer groups are further equipped with an absolute $\mathbb{Q}$-grading, refining this relative grading. As we will review in the appendix, this is given via

$$
\operatorname{gr}^{\mathbb{Q}}[\mathfrak{a}]= \begin{cases}2 k-\eta\left(D_{A}\right)+\frac{1}{4} \eta_{Y}-\frac{1}{2 \pi^{2}} C S(A) ; & \mathfrak{a}=\left(A, 0, \Phi_{k}^{A}\right) \in \mathfrak{C}^{s}, \\ -\eta\left(\widehat{\mathcal{H}}_{\left(A, s \Phi^{\prime}\right)}\right)+\frac{5}{4} \eta_{Y}-\frac{1}{2 \pi^{2}} C S(A) ; & \mathfrak{a}=(A, s, \Phi) \in \mathfrak{C}^{o}, s \neq 0 .\end{cases}
$$


where $\Phi_{k}^{A}$ above denotes the $k$ th positive eigenvector of $D_{A}$ (see Sect. 1), and $\eta_{Y}$ and $\eta_{D_{A}}$ denote the eta invariant of the corresponding operator, which we will review in Sect. 3.

\section{3 $\mathrm{ECH}=\mathrm{HM}$}

We now state the isomorphism between the ECH and HM, proved in [41]. Given a contact manifold $\left(Y^{3}, \lambda\right)$ with $d \lambda$-compatible almost complex structure $J$ as before, we define a metric $g^{T Y}$ via $\left.g^{T Y}\right|_{\xi}=d \lambda(., J),.|R|=1$ and $R$ and $\xi$ are orthogonal. This metric is adapted to the contact form in the sense $* d \lambda=2 \lambda,|\lambda|=1$. Decompose $\xi \otimes$ $\mathbb{C}=\underbrace{K}_{\xi 1,0} \oplus \underbrace{K^{-1}}_{\xi^{0,1}}$ into the $i,-i$ eigenspaces of $J$. The contact structure now determines the canonical spin-c structure $\mathfrak{s}^{\xi}$ via $S^{\xi}=\mathbb{C} \oplus K^{-1}$ with Clifford multiplication $c^{\xi}$ given by

$$
\begin{aligned}
& c^{\xi}(R)=\left[\begin{array}{ll}
i & \\
& -i
\end{array}\right], \\
& c^{\xi}(v)=\left[v^{0,1}{ }^{-i_{v^{1,0}}}\right], \quad v \in \xi .
\end{aligned}
$$

Furthermore, there is a unique spin-c connection $A_{c}$ on $S^{\xi}$ with the property that $D_{A_{c}}\left[\begin{array}{l}1 \\ 0\end{array}\right]=0$ and we call the induced connection $A_{c}^{t}$ on $K^{-1}=\operatorname{det}\left(S^{\xi}\right)$ the canonical connection. Tensor product with an auxiliary Hermitian line bundle $E$ via $S^{E}=S^{\xi} \otimes E$ and $c^{E}=c^{\xi} \otimes 1$ gives all other spin-c structures $\mathfrak{s}^{E}$. Furthermore all spin-c connections on $S^{E}$ arise as $A=A_{c} \otimes 1+1 \otimes \nabla^{A}$ for some unitary connection $\nabla^{A}$ on $E$. The $\mathrm{ECH} / \mathrm{HM}$ isomorphism is then

$$
\overline{H M}_{*}\left(-Y, \mathfrak{s}^{E}\right)=E C H_{*}\left(Y, \xi ; \text { P.D. } c_{1}(E)\right) \text {. }
$$

In the literature, this isomorphism is often stated with the left hand side given by the cohomology group $\widehat{H M}^{*}(Y)$ instead; the point is that $\widehat{H M}^{*}(Y)$ and $\widehat{H M}(-Y)$ are canonically isomorphic, see [26, Sect. 22.5, Proposition 28.3.4]. The isomorphism (2.6) allows us to define a $\mathbb{Q}$-grading on $\mathrm{ECH}$, by declaring that (2.6) preserves this Q-grading.

We now state the main ideas involved in the isomorphism (we restrict attention to the case when $c_{1}\left(\operatorname{det} \mathfrak{s}^{E}\right)$ is torsion, which is the case which is relevant here, and we sometimes state estimates that, while true, are stronger than those originally proved by Taubes). To this end, let $\sigma \in \overline{H M}\left(-Y, \mathfrak{s}^{E}\right)$. We use the perturbed Chern-SimonsDirac functional (2.2) and its gradient flow (2.3) with $\mu=\operatorname{ir} \lambda, r \in[0, \infty)$, in defining monopole Floer homology. (One also adds a small term $\eta$ to $\mu$ to achieve transversality, see for example [16], but to simplify the notation we will for now suppress this term.) Giving a family of (isomorphic) monopole Floer groups $\overline{H M}\left(-Y, \mathfrak{s}^{E}\right)$, the class $\sigma$ is hence representable by a formal sum of solutions to (2.3) corresponding to $\mu=\operatorname{ir} \lambda$. 
Denote by $\overleftarrow{C}^{r}$ the $\mu=\operatorname{ir} \lambda$ version of the complex $\widetilde{C}$ and note that its reducible generators are all of the form $\mathfrak{a}=\left(A, 0, \Phi_{k}\right)$ where $A=A_{0}-i r \lambda, A_{0}^{t}$ is flat and $\Phi_{k}$ is the $k$ th positive eigenvector of $D_{A}$. An important estimate $\eta\left(D_{A_{0}-i r \lambda}\right)=O(r)$ now gives that the grading of this generator $\operatorname{gr}^{\mathbb{Q}}[\mathfrak{a}]=\frac{r^{2}}{4 \pi^{2}} \int \lambda \wedge d \lambda+O(r)>\operatorname{gr}^{\mathbb{Q}}[\sigma]$ by (2.5) for $r \gg 0$. Hence for $r \gg 0$ the class $\sigma$ is represented by a formal sum of irreducible solutions to (2.3) with $\mu=i r \lambda$, and by a max-min argument, we may choose a family $\left(A_{r}, \Psi_{r}\right):=\left(A_{r}, s_{r} \Phi_{r}\right)$ satisfying

$$
\operatorname{gr}^{\mathbb{Q}}[\sigma]=\operatorname{gr}^{\mathbb{Q}}\left[\left(A_{r}, \Psi_{r}\right)\right]
$$

Following a priori estimates on solutions to the Seiberg-Witten equations, one then proves another important estimate $\eta\left(\widehat{\mathcal{H}}_{\left(A_{r}, \Psi_{r}\right)}\right)=O\left(r^{3 / 2}\right)$ uniformly in the class $\sigma$. This gives $C S\left(A_{r}\right)=O\left(r^{3 / 2}\right)$ which in turn by a differential relation (see Sect. 4) leads to $e_{\lambda}\left(A_{r}\right)=O(1)$. The final step in the proof shows that for any sequence of solutions $\left(A_{r}, \Psi_{r}\right)$ to Seiberg-Witten equations with $e_{\lambda}\left(A_{r}\right)$ bounded, the $E$ component $\Psi_{r}^{+}$of the spinor $\Psi_{r}=\left[\begin{array}{c}\Psi_{r}^{+} \\ \Psi_{r}^{-}\end{array}\right] \in C^{\infty}(Y ; \underbrace{E \oplus K^{-1} E}_{=S^{E}})$ satisfies the weak convergence $\left(\Psi_{r}^{+}\right)^{-1}(0) \rightarrow\left\{\left(\alpha_{j}, m_{j}\right)\right\}$ to some ECH orbit set. This last orbit set is what corresponds to the image of $\sigma \in \overline{H M}\left(Y, \mathfrak{s}^{E}\right)$ in ECH under the isomorphism (2.6). Furthermore, crucially for our purposes, one has

$$
c_{\sigma}(\lambda)=\lim _{r \rightarrow \infty} \frac{e_{\lambda}\left(A_{r}\right)}{2 \pi}
$$

see [16, Proposition 2.6]. (The proof in [16, Proposition 2.6] is given in the case where $\lambda$ is nondegenerate, but it holds for all $\lambda$ by continuity.)

\section{Estimating the eta invariant}

Let $D$ be a generalized Dirac operator acting on sections of a Clifford bundle $E$ over a closed, oriented Riemannian manifold $Y$. Then the sum

$$
\eta(D, s):=\sum_{\lambda \neq 0} \frac{\operatorname{sgn}(\lambda)}{|\lambda|^{s}}
$$

is a convergent analytic function of a complex variable $s$, as long as $\operatorname{Re}(s)$ is sufficiently large; here, the sum is over the nonzero eigenvalues of $D$. Moreover, the function $\eta(D, s)$ has an analytic continuation to a meromorphic function on $\mathbb{C}$ of $s$, which we also denote by $\eta(D, s)$, and which is holomorphic near 0 . We now define

$$
\eta(D):=\eta(D, 0) .
$$


We should think of this as a formal signature of $D$, which we call the eta invariant of Atiyah-Patodi-Singer [3].

We will be primarily concerned with the case where $D=D_{A_{r}}$, namely $D$ is the spin-c Dirac operator for a connection $A_{r}$ solving (2.3). Another case of interest to us is where $D$ is the odd signature operator on $C^{\infty}\left(Y ; i T^{*} Y \oplus \mathbb{R}\right)$ sending

$$
(a, f) \mapsto\left(* d a-d f, d^{*} a\right)
$$

in which case we denote the corresponding $\eta$ invariant by $\eta_{Y}$.

Now consider the Seiberg-Witten equations (2.3) corresponding to $\mu=i r \lambda$, for a torsion $\operatorname{spin}^{c}$ structure as above, and note that an irreducible solution (after rescaling the spinor) corresponds to a solution $\left(A_{r}, \Psi_{r}\right)$ to the Seiberg-Witten equations on $\mathcal{C}(Y, \mathfrak{s})$ given via

$$
\begin{aligned}
\frac{1}{2} c\left(* F_{A^{t}}\right)+r\left(\Psi \Psi^{*}\right)_{0}+c(\operatorname{ir} \lambda) & =0 \\
D_{A} \Psi & =0 .
\end{aligned}
$$

A further small perturbation is needed to obtain transversality of solutions see [16, Sect. 2.1]. We ignore these perturbation as they make no difference to the overall argument.

We can now state the primary result of this section:

Proposition 5 Any solution to (3.2) satisfies $\eta\left(\widehat{\mathcal{H}}_{\left(A_{r}, \Psi_{r}\right)}\right)=O\left(r^{\frac{3}{2}}\right)$.

The purpose of the rest of the section will be to prove this.

\subsection{Known estimates}

We first collect some known estimates on solutions to the equations (3.2).

Lemma 6 For some constants $c_{q}, q=0,1,2, \ldots$, we have

$$
\left|\nabla^{q} F_{A^{t}}\right| \leq c_{q}\left(1+r^{1+q / 2}\right)
$$

Proof We first note that we have the estimates:

$$
\begin{aligned}
\left|\Psi_{r}^{+}\right| & \leq 1+\frac{c_{0}}{r} \\
\left|\Psi_{r}^{-}\right| & \leq \frac{c_{0}}{r}\left(\left.|1-| \Psi_{r}^{+}\right|^{2} \mid+\frac{1}{r}\right) \\
\left|\left(\nabla^{A}\right)^{q} \Psi_{r}^{+}\right| & \leq c_{q}\left(1+r^{q / 2}\right) \\
\left|\left(\nabla^{A}\right)^{q} \Psi_{r}^{-}\right| & \leq c_{q}\left(1+r^{(q-1) / 2}\right)
\end{aligned}
$$


The first two of these estimates are proved in [40, Lemma 2.2]. The third and fourth are proved in [40, Lemma 2.3].

The lemma now follows by combining the above estimates with the equation (3.2).

In (4), we will also need:

Lemma 7 One has the bound

$$
\left|C S\left(A_{r}\right)\right| \leq c_{0} r^{2 / 3} e_{\lambda}\left(A_{r}\right)^{4 / 3}
$$

where the constant $c_{0}$ only depends on the metric contact manifold.

Proof This is proven in [40, Eq. 4.9], see also [16, Lemma 2.7].

\subsection{The $\eta$ invariant of families of Dirac operators}

In this section, we prove the key Proposition 5. The main point that we need is the following fact concerning the $\eta$ invariant:

Proposition 8 Let $A_{r}$ be a solution to (3.2). Then $\eta\left(D_{A_{r}}\right)$ is $O\left(r^{3 / 2}\right)$ as $r \rightarrow \infty$.

Before giving the proof, we first explain our strategy.

The first point is that we have the following integral formula for the $\eta$ invariant:

$$
\eta\left(D_{A_{r}}\right)=\int_{0}^{\infty} \frac{1}{\sqrt{\pi t}} \operatorname{tr}\left(D_{A_{r}} e^{-t D_{A_{r}}^{2}}\right) d t
$$

where the right hand side is a convergent integral. This is proved in [8, Sect. 2], by Mellin transform it is equivalent to the fact that the eta function $\eta\left(D_{A_{r}}, s\right)$ in (3.1) is holomorphic for $\operatorname{Re}(s)>-2$.

We therefore have to estimate the integral in (3.5). To do this, we will need the following estimates:

Lemma 9 There exists a constant $c_{0}$ independent of $r$ such that for all $r \geq 1, t>0$ :

$$
\begin{gathered}
\left|\operatorname{tr}\left(D_{A_{r}} e^{-t D_{A_{r}}^{2}}\right)\right| \leq c_{0} r^{2} e^{c_{0} r t}, \quad \text { and } \\
\left|\operatorname{tr}\left(e^{-t D_{A_{r}}^{2}}\right)\right| \leq c_{0} t^{-3 / 2} e^{c_{0} r t} .
\end{gathered}
$$

Once we have proved Lemma 9, Proposition 8 will follow from a short calculation, which we will give at the end of this section.

The proof of Lemma 9 will require two auxiliary lemmas, see Lemmas 10 and 11 below, and some facts about the heat equation associated to a Dirac operator that we will now first recall. Let $D$ be a Dirac operator on a Clifford bundle $V$ over a closed manifold $Y$. The heat equation associated to $D$ is the equation

$$
\frac{\partial s}{\partial t}+D^{2} s=0
$$


for sections $s$, and nonnegative time $t$; the operator $e^{-t D^{2}}$ is the solution operator for this equation. The heat equation has an associated heat kernel $H_{t}(x, y)$ which is a (time-dependent) section of the bundle $V \otimes V$ over $Y \times Y$ whose fiber over a point $(x, y)$ is $V_{x} \otimes V_{y}^{*}$; it is smooth for $t>0$. For any smooth section $s$ of $V$ and $t>0$, the heat kernel satisfies

$$
e^{-t D^{2}} s(x)=\int_{Y} H_{t}(x, y) s(y) \operatorname{vol}(y) .
$$

Also,

$$
\left[\frac{\partial}{\partial t}+D_{x}^{2}\right] H_{t}(x, y)=0
$$

where $D_{x}$ denotes the Dirac operator applied in the $x$ variables.

Moreover,

$$
\operatorname{tr}\left(e^{-t D^{2}}\right)=\int_{Y} \operatorname{tr}\left(H_{t}(y, y)\right) \operatorname{vol}(y) .
$$

Hence, we can prove Lemma 9 by bounding $\left|H_{t}\right|$ along the diagonal. The operator $D e^{-t D^{2}}$ has a kernel $L_{t}(x, y)$ as well, and the analogous results hold.

A final fact we will need is Duhamel's principle: this says that the inhomogeneous heat equation

$$
\frac{\partial \tilde{s}}{\partial t}+D^{2} \tilde{s}_{t}=s_{t}
$$

has a unique solution tending to 0 with $t$, given by

$$
\tilde{s}_{t}(x)=\int_{0}^{t}\left(e^{-\left(t-t^{\prime}\right) D^{2}} s_{t^{\prime}}\right)(x) d t^{\prime},
$$

as long as $s_{t}$ is a smooth section of $S$, continuous in $t$.

Now let $D$ be $D_{A_{r}}$, and $V$ the spinor bundle for the $\operatorname{spin}^{c}$ structure $S$, and let $H_{t}^{r}$ and $L_{t}^{r}$ be defined as above, but with $D=D_{A_{r}}$. Let $\rho(x, y)$ the Riemannian distance function. Define an auxiliary function

$$
h_{t}(x, y):=(4 \pi t)^{-3 / 2} e^{-\frac{\rho(x, y)^{2}}{4 t}} .
$$

In the case of $Y=\mathbb{R}^{3}$, with $\rho$ the standard Euclidean distance, the function $h_{t}(x, y)$ is precisely the ordinary heat kernel. In our case, the kernel $H_{t}^{r}(x, y)$ has an asymptotic expansion as $t \rightarrow 0$,

$$
H_{t}^{r}(x, y) \sim h_{t}(x, y)\left(b_{0}^{r}(x, y)+b_{1}^{r}(x, y) t+b_{2}^{r}(x, y) t^{2}+\cdots+\right),
$$


that is studied in detail in [7, Chap. 2]; here, the $b_{i}^{r}(x, y)$ are defined on all of $Y \times Y$. The following lemma summarizes what we need to know about the results from [7, Chap. 2]:

Lemma 10 There exists for all $i=0,1,2, \ldots$ sections $b_{i}^{r}(x, y)$ such that:

- The $b_{i}^{r}$ are supported in any neighborhood of the diagonal.

- The asymptotic expansion (4.16) may be formally differentiated to obtain asymptotic expansions for the derivative. In particular, there is an asymptotic expansion

$$
L_{t}^{r}(x, y) \sim h_{t}(x, y)\left(\tilde{b}_{0}^{r}(x, y)+\tilde{b}_{1}^{r}(x, y) t+\tilde{b}_{2}^{r}(x, y) t^{2}+\cdots+\right)
$$

where

$$
\tilde{b}_{n}^{r}(x, y)=\left(D_{A_{r}}+c(\rho d \rho / 2 t)\right) b_{n}^{r}(x, y) .
$$

- For any $n, t>0$,

$$
L_{t}^{r}(x, y)-h_{t}(x, y) \sum_{i=0}^{n} \tilde{b}_{i}^{r}(x, y) t^{i}
$$

is $O\left(t^{i-1 / 2}\right)$, in the $C^{0}$-norm on the product, as $t \rightarrow 0$.

$$
\left(\partial_{t}+D_{A_{r}}^{2}\right)\left(L_{t}^{r}(x, y)-h_{t}(x, y) \sum_{i=0}^{n} \tilde{b}_{i}^{r}(x, y) t^{i}\right)=-D_{A_{r}}^{2} \tilde{b}_{n}^{r} t^{n}
$$

Proof The lemma summarizes those parts of the proof of [7, Theorem 2.30] that we will soon need; the arguments in [7, Theorem 2.30] provide the proof. The idea behind the first bullet point is that $h_{t}(x, y)$ is on the order of $t^{\infty}$ away from the diagonal. The reason for the $i-1 / 2$ exponent in the third bullet point is that $h_{t}$ has a $t^{-3 / 2}$ term. For the fourth bullet point, the point is that the coefficients $b_{i}^{r}$ are constructed so as to satisfy (3.7) when formally differentiating (4.16) and equating powers of $t$; this gives a recursion which is relevant for our purposes because it implies that when we truncate the expansion at a finite $n$, the inhomogeneous equation (3.12) is satisfied.

In view of the first bullet point of the above lemma, we only have to understand the coefficients $b_{i}^{r}$ in a neighborhood of the diagonal. To facilitate this, let $i_{g^{T Y}}$ denote the injectivity radius of the Riemannian metric $g$, and given $y \in Y$, let $B_{y}\left(\frac{{ }{ }_{g} T Y}{2}\right)$ denote a geodesic ball of radius $\frac{i_{g} T Y}{2}$ centered at $y$, and let $y$ denote a choice of co-ordinates on this ball. Define $G_{y}^{k} \subset C^{\infty}\left(B_{y}\left(\frac{{ }_{{ }_{g} T Y}}{2}\right)\right)$ to be the subspace of ( $r$-dependent) functions $f$ satisfying the estimate $\partial_{y}^{\alpha} f=O\left(r^{k+\frac{|\alpha|}{2}}\right)$ as $r \rightarrow \infty, \forall \alpha \in \mathbb{N}_{0}^{3}$, and for 
each $j \in \frac{1}{2} \mathbb{N}_{0}$, further define the subspace $W_{y}^{j} \subset C^{\infty}\left(B_{y}\left(\frac{{ }_{{ }_{g} T Y}}{2}\right)\right)$ via

$$
f \in W_{y}^{j} \Longleftrightarrow f=\sum_{i=1}^{N} f_{i}, \quad \text { with each } f_{i} \in y^{\alpha} G_{y}^{k}, k \leq j+\frac{|\alpha|}{2} .
$$

Finally, given $y \in Y$, we choose a convenient frame for $S_{y}^{\xi}$ and $E$ over $B_{y}\left(\frac{{ }_{i_{g} T Y}}{2}\right)$, which we will call a synchronous frame; specifically, choose an orthonormal basis for each of $S_{y}^{\xi}, E_{y}$, and parallel transport along geodesics with $A_{c}, A_{r}$ to obtain local orthonormal trivializations $\left\{s_{1}, s_{2}\right\},\{e\}$. Now, if $b$ is any section of $S \otimes S_{y}$ over $B_{y}\left(\frac{i_{g} T Y}{2}\right) \times\{y\}$ write

$$
b(\cdot, y)=\sum_{k, l=1}^{2} f_{b, k l}^{y}(.)\left(s_{k} \otimes e\right)(.)\left(s_{l} \otimes e\right)^{*}(y) .
$$

Lemma 11 There is a constant $c_{0}$ independent of $r$ such that for any $t>0, r \geq 1$, we have

$$
\left|H_{t}^{r}(x, y)\right| \leq c_{0} h_{2 t}(x, y) e^{c_{0} r t}
$$

Further, for any $y \in Y$, the restriction of the terms $b_{j}^{r}$ to $B_{y}\left(\frac{i_{g} T Y}{2}\right) \times\{y\}$ have the property that their corresponding functions $f_{b_{j}^{r}, k l}^{y}$ in (3.14) are all in $W_{y}^{j}$.

Proof The first bullet point is similar to [35, Proposition 3.1].

To prove the second bullet point, we use the fact that the terms $b_{j}^{r}$ in the heat kernel expression (4.16) are known to satisfy a recursion, as alluded to above, and explained in the proof of [7, Theorem 2.30]. Specifically, fix $y \in Y$, choose geodesic coordinates $y$ around $y$, mapping 0 to $y$, and choose a synchronous frame as in (3.14). Then, in these coordinates, we have

$$
b_{0}^{r}(x, y)=\sum_{i=1}^{2} g^{-1 / 4}(x)\left(s_{i} \otimes e\right)(x)\left(s_{i} \otimes e\right)^{*}(y),
$$

where $g=\operatorname{det}\left(g_{j k}\right)$. Moreover, if use these coordinates to identify sections of $S \otimes S_{y}^{*}$ with a vector of functions, then we have

$$
b_{j}^{r}(x, y)=-\frac{1}{g^{1 / 4}(x)} \int_{0}^{1} \rho^{j-1} g^{1 / 4}(\rho x) D_{A_{r}}^{2} b_{j-1}^{r}(\rho x, y) d \rho, \quad j \geq 1
$$

where on the right hand side of this equation, we mean that we are integrating this vector of functions component by component. 
Now recall the Bochner-Lichnerowicz-Weitzenbock formula for the Dirac operator:

$$
D_{A_{r}}^{2}=\nabla_{A_{r}}^{*} \nabla_{A_{r}}+\frac{\kappa}{4}-\frac{1}{2} c\left(* F_{A_{r}}\right)
$$

where $\kappa$ denotes the scalar curvature; we will want to combine this with (3.17). In coordinates, we have

$$
\nabla_{A_{r}}=\left(\partial_{1}+\Gamma_{1}, \partial_{2}+\Gamma_{2}, \partial_{3}+\Gamma_{3}\right)
$$

where each $\Gamma_{i}$ is the $i^{\text {th }}$ Christoffel symbol for $A_{r}$. We also have

$$
\nabla_{A_{r}}^{*}=-\sum_{j, k}^{3}-g^{j k}\left(\partial_{k}+\Gamma_{k}\right)+\sum_{i, j, k}^{3} g^{j k} \Gamma_{j k}^{i},
$$

where the $\Gamma_{j, k}^{i}$ are the Christoffel symbols of the Riemannian metric. Since we have $A_{r}=1 \otimes A+A_{c} \otimes 1$, where $A_{c}$ is the canonical connection on $S^{\xi}$, we can decompose each Christoffel symbol

$$
\Gamma_{i}=c_{i}+a_{i}
$$

where the $c_{i}$ are Christoffel symbols for $A_{c}$ and the $a_{i}$ are Christoffel symbols for $A$.

The $c_{i}$ are independent of $r$. To understand the $a_{j}$, first write the defining equations for the curvature

$$
F_{k j}=\partial_{k} a_{j}-\partial_{j} a_{k}
$$

Now write the coordinate $x=\left(x^{1}, x^{2}, x^{3}\right)$, and consider $\sum_{k=1}^{3} x^{k}\left(\partial_{k} a_{j}-\partial_{j} a_{k}\right)$. Reintroducing the radial coordinate $\rho$, we have

$$
\rho \sum_{k=1}^{3} x^{k} \partial_{k} a_{j}=\frac{\partial a_{j}}{\partial \rho}
$$

On the other hand, since the frame $e$ is parallel, we have $\nabla_{x^{1}} \partial_{x_{1}}+x^{2} \partial_{x_{2}}+x^{3} \partial_{x_{3}} e=$ $\nabla_{\rho \partial_{\rho}} e=0$, hence $\sum_{k=1}^{3} x^{k} a_{k}=0$. Thus, we have

$$
a_{j}(x)=\sum_{k=1}^{3} \int_{0}^{1} d \rho \rho x^{k} F_{k j}(\rho x)
$$

In particular, it follows from the a priori estimate (3.23) and (3.22) that each

$$
a_{j} \in W_{y}^{\frac{1}{2}}
$$


Now note that we have $W_{y}^{j}+W_{y}^{k} \subset W_{y}^{\max \{j, k\}}, W_{y}^{j} \cdot W_{y}^{k} \subset W_{y}^{j+k}$, and $\partial_{y} W_{y}^{j} \subset$ $W_{y}^{j+\frac{1}{2}}$. Hence, by (3.18), (3.19), (3.20), and (3.23), we have that the square of the Dirac operator has the schematic form

$$
D_{A_{r}}^{2}=\sum_{j, k}-g^{j k} \partial_{j} \partial_{k}+P_{j} \partial_{j}+Q
$$

where $P_{j} \in W^{\frac{1}{2}}$ and $Q \in W_{y}^{1}$. The Lemma now follows by induction, using (3.16) and (3.17).

We now give the proof of Lemma 9:

Proof of Lemma 9 The second bullet point follows by combining (3.8) and (3.15).

To prove the first bullet point, our strategy will be to bound the pointwise size of the kernel $L_{t}^{r}(y, y)$ and appeal to the version of (3.8) for $L_{t}^{r}$.

To do this, consider the asymptotic expansion (3.11). By a theorem of BismutFreed ([8, Theorem 2.4]), for any $y \in Y, \operatorname{tr} L_{t}^{r}(y, y)$ is $O\left(t^{1 / 2}\right)$ as $t \rightarrow 0$. So we have $\operatorname{tr} L_{t}^{r}(y, y)=\operatorname{tr} R_{t}^{r}(y, y)$ for the remainder

$$
R_{t}^{r}(x, y):=L_{t}^{r}-D_{A_{r}}\left[h_{t}\left(b_{0}+t b_{1}\right)\right]
$$

By (3.12), $R_{t}^{r}$ satisfies the inhomogeneous heat equation

$$
\left(\partial_{t}+D_{A_{r}}^{2}\right) R_{t}^{r}(x, y)=h_{t} t\left\{-D_{A_{r}}^{3} b_{1}+c\left(\frac{\rho d \rho}{2 t}\right) D_{A_{r}}^{2} b_{1}\right\},
$$

and by the third bullet point of Lemma $10, R_{t}^{r} \rightarrow 0$ as $t \rightarrow 0$. We can then apply Duhamel's principle (3.9) to write

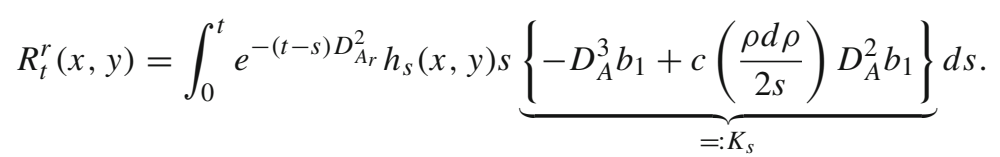

We can then apply the key property of the heat kernel (3.6) to write

$$
R_{t}^{r}(x, y)=\int_{0}^{t} \int_{Y} H_{t-s}^{r}(x, z) h_{s}(z, y) s K_{s}(z, y) \operatorname{vol}(z) d s,
$$

and we can apply the second bullet point of Lemma 10 to conclude that

$$
\left|R_{t}^{r}(y, y)\right| \leq c_{0} \int_{0}^{t} \int_{Y} e^{c_{0} r(t-s)} h_{s}(z, y) h_{2(t-s)}(z, y) s K_{s}(z, y) \operatorname{vol}(z) d s .
$$


By the first bullet point of Lemma 10, we can assume that $K_{S}(z, y)$ is supported in $B_{y}\left(\frac{i_{g} T Y}{2}\right) \times\{y\}$. Thus, we just have to bound

$$
\int_{0}^{t} \int_{B_{y}\left(\frac{{ }_{g} T Y}{2}\right)} e^{c_{0} r(t-s)} h_{s}(y, 0) h_{2(t-s)}(y, 0) s K_{s}(y, 0) d y d s,
$$

where $y$ are geodesic coordinates centered at $y$. To do this, choose a synchronous frame for the spinor bundle, as we have been doing above. Then, following (3.21), (3.22), in these coordinates the Dirac operator is seen to have the form

$$
D_{A_{r}}=w^{j k} \partial_{j}+K
$$

for $r$-independent $w^{j k}$ and $K \in W_{y}^{\frac{1}{2}}$, in the geodesic coordinates and orthonormal frame introduced before. Combining this with the second bullet point of Lemma 11 gives that the term $K_{s} \in W_{y}^{\frac{5}{2}}$. So, (3.26) is dominated by a finite sum of integrals of the form

$$
\int_{0}^{t} d s \int_{B_{y}\left(\frac{{ }_{g}{ }_{g} T Y}{2}\right)} d y s e^{c_{0} r t} h_{2(t-s)}(y, 0) h_{s}(y, 0) y^{\alpha} r^{k}, \quad k \leq \frac{5}{2}+\frac{|\alpha|}{2} .
$$

On $B_{y}\left(\frac{i_{g} T Y}{2}\right)$, we have

$$
y^{I} h_{t}(y, 0) \leq c_{1} t^{\frac{1}{2}|I|} h_{2 t}(y, 0)
$$

for some constant $c_{1}$. Hence, we can bound the above integral by

$$
c_{1} r^{k} e^{c_{0} r t} \int_{0}^{t} d s \int_{B_{y}\left(\frac{{ }_{g}{ }_{g} T Y}{2}\right)} d y s^{1+\frac{|\alpha|}{2}} h_{2(t-s)}(y, 0) h_{2 s}(y, 0) d y .
$$

We also have

$$
\int_{Y} h_{t}(x, y) h_{t^{\prime}}(y, z) d y \leq c_{2} h_{4\left(t+t^{\prime}\right)}(x, z)
$$

as proved in [35, Sect. A]. So, we can bound (3.27) by

$$
c_{3} r^{k} e^{c_{0} r t} \int_{0}^{t} s^{1+\frac{|\alpha|}{2}} h_{8 t}(0,0) d s=c_{3} r^{k} e^{c_{0} r t} \int_{0}^{t} s^{1+\frac{|\alpha|}{2}}(4 t)^{-3 / 2} d s \leq c_{3} r^{k} t^{\frac{1+|\alpha|}{2}} e^{c_{0} r t} .
$$

We know that $k-\frac{1+|\alpha|}{2} \leq 2$. So, putting all of the above together, (3.26) is dominated by a finite sum of terms of the form

$$
r^{2} r^{\frac{1+|\alpha|}{2}} t^{\frac{1+|\alpha|}{2}} e^{c_{0} r t}
$$


which proves the result.

We can finally give the proof of Proposition 8.

Proof of Proposition 8 Define $E(x):=\operatorname{sign}(x) \operatorname{erfc}(|x|)=\operatorname{sign}(x) \cdot \frac{2}{\sqrt{\pi}} \int_{|x|}^{\infty} e^{-s^{2}} d s<$ $e^{-x^{2}}$. This is a rapidly decaying function, so the function $E\left(D_{A_{r}}\right)$ is defined, and its trace is a convergent sum

$$
\operatorname{tr}\left(E\left(D_{A_{r}}\right)\right)=\sum_{\lambda} E(\lambda)
$$

where $\lambda$ is an eigenvalue of $D_{A_{r}}$. The eta invariant in unchanged under positive rescaling

$$
\eta\left(D_{A_{r}}\right)=\eta\left(\frac{1}{\sqrt{r}} D_{A_{r}}\right)
$$

Now use (3.5) to rewrite the right hand side of the above equation as

$$
\left|\int_{0}^{1} d t \frac{1}{\sqrt{\pi t}} \operatorname{tr}\left[\frac{1}{\sqrt{r}} D_{A_{r}} e^{-\frac{t}{r} D_{A_{r}}^{2}}\right]+\operatorname{tr} E\left(\frac{1}{\sqrt{r}} D_{A_{r}}\right)\right| .
$$

The absolute value of the first summand in the above expression is bounded from above by a constant multiple of $r^{3 / 2}$, by the first bullet point in Lemma 9 . The absolute value of the second summand in the same expression is bounded from above by $\operatorname{tr} e^{-\frac{1}{r} D_{A_{r}}^{2}}$, which by the second bullet point in Lemma 9 is bounded by a constant multiple of $r^{3 / 2}$ as well.

Proof of Proposition 5 An application of the Atiyah-Patodi-Singer index theorem as in Sect. 1 gives

$$
\frac{1}{2} \eta\left(\widehat{\mathcal{H}}_{\left(A_{r}, \Psi_{r}\right)}\right)=\frac{1}{2} \eta\left(D_{A_{r}}\right)+\frac{1}{2} \eta_{Y}+\operatorname{sf}\left\{\widehat{\mathcal{H}}_{\left(A_{r}, \epsilon \Psi_{r}\right)}\right\}_{0 \leq \varepsilon \leq 1}
$$

The spectral flow term above is estimated to be $O\left(r^{3 / 2}\right)$ as in [40, Sect. 5.4] while $\eta\left(D_{A_{r}}\right)=O\left(r^{3 / 2}\right)$ by Proposition 8 .

We also note that the constant in Proposition 5 above is only a function of $(Y, \lambda, J)$ and independent of the class $\sigma=\left[\left(A_{r}, \Psi_{r}\right)\right] \in \overline{H M}\left(-Y, \mathfrak{s}^{E}\right)$ defined by the SeibergWitten solution.

Remark 12 The reason that we can not improve upon $\mathrm{gr}^{\mathbb{Q}}$ asymptotics is because we do not know how to strengthen the $O\left(r^{3 / 2}\right)$ spectral flow estimate on the irreducible solutions of Propositions 5 or 8 . A better $O(r)$ estimate does however exist [35,37] for reducible solutions for which one understands the connection precisely in the limit $r \rightarrow \infty$. However, the a priori estimates (3.23) are not strong enough to carry out the same for irreducibles. 


\section{Asymptotics of capacities}

\subsection{The main theorem}

In this section we now prove our main theorem Theorem 2 on ECH capacities.

Proof of Theorem 2 Let $0 \neq \sigma_{j} \in E C H(Y, \lambda, \Gamma), j=0,1,2, \ldots$, be a sequence of non-vanishing classes with gradings

$$
\operatorname{gr}^{\mathbb{Q}}\left(\sigma_{j}\right)=q+j
$$

$j \in 2 \mathbb{N}$. As in Sect. 2.3, we use the perturbed Chern-Simons-Dirac functional (2.2) $\mathcal{L}_{\mu}$ and its gradient flow (2.3) with $\mu=i r \lambda, r \in[0, \infty)$, in defining monopole Floer homology. Hence for each $r \in[1, \infty)$, the class $\sigma_{j}$ may be represented by a formal sum of solutions to (2.3) with $\mu=i r \lambda$. As noted in Sect. 2.3, this solution is eventually irreducible.

We now estimate $r_{1}(j)$, the infimum of the values of $r$ such that each solution $\left[\mathfrak{a}_{j}\right]_{r}$ to (2.3) representing $\sigma_{j}$ is irreducible. For this note that a reducible solution is of the form $\mathfrak{a}=\left(A, 0, \Phi_{k}\right)$ where $A=A_{0}-i r \lambda, A_{0}^{t}$ flat and $\Phi_{k}$ the $k$ th positive eigenvector of $D_{A}$. The grading of such a reducible is given by (2.5). The important estimate $\left|\eta_{D_{A_{1}+r \lambda}}\right| \leq c_{0} r,\left(\left[36\right.\right.$, Theorem 1.2]) now shows gr $\mathbb{Q}\left[\left(A, 0, \Phi_{k}\right)\right]>\operatorname{gr}^{\mathbb{Q}}\left(\sigma_{j}\right)=q+j$ for

$$
r>\bar{r}_{1}(j):=\sup \left\{r \mid \frac{r^{2}}{4 \pi^{2}} \operatorname{vol}(Y, \lambda)<c_{0} r+q+j\right\}
$$

Hence $r_{1}(j)<\bar{r}_{1}(j)$. Furthermore

$$
\bar{r}_{1}(j)=2 \pi\left[\frac{j}{\operatorname{vol}(Y, \lambda)}\right]^{1 / 2}+O(1) \text { as } j \rightarrow \infty
$$

from the above definition. A max-min argument, as also mentioned in Sect. 2.3 then gives $\forall j \in 2 \mathbb{N}$ a piecewise-smooth family of irreducible solutions $[\mathfrak{a}]_{r}=\left(A_{r}, \Psi_{r}\right)$, $r>r_{1}(j)$, of fixed grading $\mathrm{gr}^{\mathbb{Q}}[\mathfrak{a}]=q+j$ such that $\mathcal{L}_{\mu}$ is continuous, see (for example) [16, Sect. 2.6].

By (3.4), we have

$$
|C S(A)| \leq c_{0} r^{2 / 3} e_{\lambda}(A)^{4 / 3}
$$

In addition, by combining (2.5) and Proposition 8, we have

$$
\left|\frac{1}{2 \pi^{2}} C S\left(A_{r}\right)-(q+j)\right| \leq c_{0} r^{3 / 2}
$$


with the constant $c_{0}>0$ being independent of the grading $j$. We also have the differential relation

$$
r \frac{d e_{\lambda}}{d r}=\frac{d C S}{d r}
$$

between the two functionals, away from the discrete set of points where derivatives are undefined, see [16, Lemma 2.5]. Now define $F(r)=\frac{1}{2} r_{1}^{2} \operatorname{vol}(Y, \lambda)+\int_{r_{1}}^{r} e_{\lambda}\left(A_{s}\right) d s$. This is a continuous function so we may integrate the above equation from $r_{1}$ to $r$ to conclude that

$$
C S(r)=r F^{\prime}-F
$$

valid for all $r$ away from the above discrete set; here, we have used [43, Property 2.3.(i)], together with the computation in [16, Lemma 2.3] in the computation of the terms at $r_{1}$.

On account of (4.3), $F$ is then a super/subsolution to the ODEs

$$
-c_{2} r^{3 / 2} \leq r F^{\prime}-F-(q+j) \leq c_{2} r^{3 / 2}
$$

for $r \geq r_{1}$. This gives

$$
\begin{array}{r}
\frac{1}{2} r r_{1} \operatorname{vol}(Y, \lambda)+r\left[\frac{q+j}{r_{1}}-\frac{q+j}{r}-2 c_{2} r^{1 / 2}+2 c_{2} r_{1}^{1 / 2}\right] \leq F \\
\frac{1}{2} r r_{1} \operatorname{vol}(Y, \lambda)+r\left[\frac{q+j}{r_{1}}-\frac{q+j}{r}+2 c_{2} r^{1 / 2}-2 c_{2} r_{1}^{1 / 2}\right] \geq F \\
\frac{1}{2} r_{1} \operatorname{vol}(Y, \lambda)+\frac{q+j}{r_{1}}-3 c_{2} r^{1 / 2}+2 c_{2} r_{1}^{1 / 2} \leq F^{\prime} \\
\frac{1}{2} r_{1} \operatorname{vol}(Y, \lambda)+\frac{q+j}{r_{1}}+3 c_{2} r^{1 / 2}-2 c_{2} r_{1}^{1 / 2} \geq F^{\prime} .
\end{array}
$$

Next the estimate (4.2) in terms of $F$ is

$$
-c_{1} r^{2 / 3}\left(F^{\prime}\right)^{4 / 3} \leq r F^{\prime}-F \leq c_{1} r^{2 / 3}\left(F^{\prime}\right)^{4 / 3}
$$

We let $\rho_{0}$ be the smallest positive root of $\frac{1}{3}-\left[\rho+\rho^{2}+\rho^{3}+\rho^{4}\right]=0$ and define

$$
\bar{r}_{2}(j)=\sup \left\{r \mid c_{1} r^{-2 / 3} F^{1 / 3} \geq \rho_{0}\right\}
$$


which is finite on account of (4.4). Further with $c_{3}=1+3\left(\frac{2 c_{1}}{3}\right)+3\left(\frac{2 c_{1}}{3}\right)^{2}$ define

$$
\begin{aligned}
\tilde{r}_{2}(j) & =\sup \left\{r \mid \frac{1}{2} r_{1} \operatorname{vol}(Y, \lambda)+\frac{q+j}{r_{1}}+3 c_{2} r^{1 / 2}-2 c_{2} r_{1}^{1 / 2} \geq\left(\frac{3}{4 c_{1}}\right)^{3} r\right. \\
& \text { or } \frac{1}{2} r_{1} \operatorname{vol}(Y, \lambda)+\frac{q+j}{r_{1}}-\frac{q+j}{r}+2 c_{2} r^{1 / 2}-2 c_{2} r_{1}^{1 / 2} \geq\left(\frac{1}{9 c_{3}}\right)^{3} r \\
& \text { or } \left.\frac{1}{2} r_{1} \operatorname{vol}(Y, \lambda)+\frac{q+j}{r_{1}}-\frac{q+j}{r}+2 c_{2} r^{1 / 2}-2 c_{2} r_{1}^{1 / 2} \geq r\right\}
\end{aligned}
$$

and set $r_{2}(j):=\max \left\{\bar{r}_{2}(j), \tilde{r}_{2}(j)\right\}$. We note that $r_{2}(j)=O\left(j^{1 / 2}\right)$. We now have the following lemma.

Lemma 13 For $r>r_{2}(j)$ we have

$$
\left(\frac{F}{r}\right)^{1 / 3}-\frac{2 c_{1}}{3 r} F^{2 / 3} \leq\left(F^{\prime}\right)^{1 / 3} \leq\left(\frac{F}{r}\right)^{1 / 3}+\frac{2 c_{1}}{3 r} F^{2 / 3}
$$

Proof By definition,

$$
\begin{aligned}
r & >r_{2}(j) \geq \bar{r}_{2}(j) \\
& \Longrightarrow \rho:=c_{1} r^{-2 / 3} F^{1 / 3}<\rho_{0} \\
& \Longrightarrow \rho+\rho^{2}+\rho^{3}+\rho^{4}<\frac{1}{3}
\end{aligned}
$$

as well as

$$
\begin{aligned}
r> & r_{2}(j) \geq \tilde{r}_{2}(j) \\
& \Longrightarrow F^{\prime} \leq \frac{1}{2 r} r_{1}^{2} \operatorname{vol}(Y, \lambda)+\frac{q+j}{r_{1}}+3 c_{2} r^{1 / 2}-2 c_{2} r_{1}^{1 / 2}<\left(\frac{3}{4 c_{1}}\right)^{3} r
\end{aligned}
$$

by (4.4).

For $y=\left(F^{\prime}\right)^{1 / 3}$ equations (4.5) become the pair of quartic inequalities

$$
\begin{aligned}
& 0 \leq c_{1} r^{-1 / 3} y^{4}-y^{3}+r^{-1} F \\
& 0 \leq c_{1} r^{-1 / 3} y^{4}+y^{3}-r^{-1} F
\end{aligned}
$$

With $y_{0}^{ \pm}=\left(\frac{F}{r}\right)^{1 / 3} \pm \frac{2 c_{1}}{3 r} F^{2 / 3}$ we calculate

$$
\begin{aligned}
& c_{1} r^{-1 / 3}\left(y_{0}^{+}\right)^{4}-\left(y_{0}^{+}\right)^{3}+r^{-1} F \\
& \quad=-r^{-5 / 3} c_{1} F^{4 / 3}\left[1-\frac{4}{3} \rho-\frac{64}{27} \rho^{2}-\frac{32}{27} \rho^{3}-\frac{16}{81} \rho^{4}\right]<0 \text { and }
\end{aligned}
$$




$$
\begin{aligned}
& c_{1} r^{-1 / 3}\left(y_{0}^{-}\right)^{4}+\left(y_{0}^{-}\right)^{3}-r^{-1} F \\
& \quad=r^{-5 / 3} c_{1} F^{4 / 3}\left[-1-\frac{4}{3} \rho+\frac{64}{27} \rho^{2}-\frac{32}{27} \rho^{3}+\frac{16}{81} \rho^{4}\right]<0 .
\end{aligned}
$$

The minimum of the quartic in (4.10), attained at $y_{\min }=\frac{3}{4 c_{1}} r^{1 / 3}$, is thus also negative. Furthermore the quartic having an inflection point at zero cannot change sign between $y_{0}^{+}, y_{\min }$. Thus (4.5) gives $\left(F^{\prime}\right)^{1 / 3}=y \leq y_{0}^{+}$or $\left(F^{\prime}\right)^{1 / 3}=y \geq y_{\min }=\frac{3}{4 c_{1}} r^{1 / 3}$. The second possibility being disallowed on account of (4.9), gives the desired upper bound of (4.8). Similarly, the minimum of quartic (4.11) is attained at the negative $y_{\min }=-\frac{3}{4 c_{1}} r^{1 / 3}$. Hence $y_{0}^{-} \leq y=\left(F^{\prime}\right)^{1 / 3}$ which is the lower bound in (4.8).

Next cubing (4.8) estimates $F^{\prime}$ as a sum the terms $\frac{F}{r}, \frac{F^{4 / 3}}{r^{5 / 3}}, \frac{F^{5 / 3}}{r^{7 / 3}}, \frac{F^{2}}{r^{3}}$. The last two $\frac{F^{5 / 3}}{r^{7 / 3}}, \frac{F^{2}}{r^{3}}$ in turn controlled by $\frac{F^{4 / 3}}{r^{5 / 3}}$ using (4.6) gives

$$
\frac{F}{r}-c_{3} \frac{F^{4 / 3}}{r^{5 / 3}} \leq F^{\prime} \leq \frac{F}{r}+c_{3} \frac{F^{4 / 3}}{r^{5 / 3}}
$$

for $r \geq r_{2}(j)$. This gives

$$
\begin{gathered}
r^{1 / 3}\left[\frac{r^{1 / 3}}{-c_{3}+c_{0}^{-} r^{1 / 3}}\right] \leq F^{1 / 3} \leq r^{1 / 3}\left[\frac{r^{1 / 3}}{c_{3}+c_{0}^{+} r^{1 / 3}}\right], \quad \text { where } \\
c_{0}^{ \pm}=\frac{r_{3}^{1 / 3} \mp c_{3}\left[\frac{F\left(r_{3}\right)}{r_{3}}\right]^{1 / 3}}{F\left(r_{3}\right)^{1 / 3}}
\end{gathered}
$$

for $r \geq r_{3}(j) \geq r_{2}(j)$. The last equation with (4.12) gives

$$
\frac{F\left(r_{3}\right)}{\left(r_{3}^{1 / 3}+c_{3}\left[\frac{F\left(r_{3}\right)}{r_{3}}\right]^{1 / 3}\right)^{3}}=\frac{1}{\left(c_{0}^{-}\right)^{3}} \leq \underbrace{F^{\prime}(r)}_{=e_{\lambda}\left(A_{r}\right)} \leq \frac{1}{\left(c_{0}^{+}\right)^{3}}=\frac{F\left(r_{3}\right)}{\left(r_{3}^{1 / 3}-c_{3}\left[\frac{F\left(r_{3}\right)}{r_{3}}\right]^{1 / 3}\right)^{3}}
$$

and hence

$$
\frac{F\left(r_{3}\right)}{r_{3}}\left[1-\frac{4 c_{3}}{r_{3}^{1 / 3}}\left(\frac{F\left(r_{3}\right)}{r_{3}}\right)^{1 / 3}\right] \leq e_{\lambda}\left(A_{r}\right) \leq \frac{F\left(r_{3}\right)}{r_{3}}\left[1+\frac{4 c_{3}}{r_{3}^{1 / 3}}\left(\frac{F\left(r_{3}\right)}{r_{3}}\right)^{1 / 3}\right]
$$

from (4.7) for $r \gg 0$. However, (4.4) for $r=r_{3}$ when substituted into the last equation above becomes

$$
\begin{aligned}
& {\left[\frac{q+j}{r_{1}}-\frac{q+j}{r_{3}}-2 c_{2} r_{3}^{1 / 2}\right](1-R)} \\
& \quad \leq e_{\lambda}\left(A_{r}\right) \leq\left[\frac{1}{2} r_{1} \operatorname{vol}(Y, \lambda)+\frac{q+j}{r_{1}}+2 c_{2} r_{3}^{1 / 2}\right](1+R)
\end{aligned}
$$




$$
\text { with } R:=\frac{4 c_{3}}{r_{3}^{1 / 3}}\left[\frac{1}{2} r_{1} \operatorname{vol}(Y, \lambda)+\frac{q+j}{r_{1}}+2 c_{2} r_{3}^{1 / 2}\right]^{1 / 3} \text {. }
$$

Setting $r_{3}=j^{4 / 5}$ (satisfying $r_{3} \geq r_{2}=O\left(j^{1 / 2}\right)$ for $j \gg 0$ ) and using (2.7), (4.1) gives

$$
c_{\sigma_{j}}(\lambda)=j^{\frac{1}{2}} \operatorname{vol}(Y, \lambda)^{\frac{1}{2}}+O\left(j^{2 / 5}\right)
$$

as $j \rightarrow \infty$, which is our main result Theorem 2 .

Remark 14 One could replace the arguments in this subsection with the arguments in Sun's paper [43], if desired-the key reason why we have a stronger bound than Sun is because of our stronger bound on the Chern-Simons functional, and not because of anything we do in this subsection. We have chosen to include our argument here, which we developed independently of the arguments in [43], for completeness, and because it might be of independent interest, although we emphasize that we do use the result of Sun establishing [43, Property 2.3.(i)].

On the other hand, the arguments in [16] are not quite strong enough for Theorem 2 , even with the improved bound in Proposition 8.

\subsection{Proofs of corollaries}

Here we prove the two corollaries Corollaries 3 and 4, both following immediately from the capacity formula Theorem 2 .

Proof of Corollary 3 The $\mathbb{Z}_{2}$ vector space $\operatorname{ECH}\left(Y, \xi, \Gamma ; \mathbb{Z}_{2}\right)$ is known to be twoperiodic, and nontrivial, in sufficiently high grading, see for example [22]. Thus, for $* \gg 0$ sufficiently large there exists a finite set of classes $\left\{\sigma_{1}, \ldots, \sigma_{2^{d}-1}\right\} \subset$ $E C H_{*}\left(Y, \xi, \Gamma ; \mathbb{Z}_{2}\right) \cup E C H_{*+1}\left(Y, \xi, \Gamma ; \mathbb{Z}_{2}\right)$ such that

$$
\begin{aligned}
& \left\{0, U^{-j} \sigma_{1}, \ldots, U^{-j} \sigma_{2^{d}-1}\right\} \\
& \quad=E C H_{*+2 j}\left(Y, \xi, \Gamma ; \mathbb{Z}_{2}\right) \cup E C H_{*+1+2 j}\left(Y, \xi, \Gamma ; \mathbb{Z}_{2}\right), \forall j \geq 0 .
\end{aligned}
$$

Thus the ECH spectrum modulo a finite set is given by

$$
\cup_{j=0}^{\infty}\left\{c_{U^{-j} \sigma_{1}}(\lambda), \ldots, c_{U^{-j} \sigma_{2^{d}-1}}(\lambda)\right\}
$$

The corollary now follows as $c_{U^{-j} \sigma_{l}}(\lambda)=j^{\frac{1}{2}} \operatorname{vol}(Y, \lambda)^{\frac{1}{2}}+O\left(j^{2 / 5}\right), 1 \leq l \leq 2^{d}-1$, by Theorem 2 .

Proof of Corollary 4 As in the previous corollary, the ECH zeta function is given, modulo a finite and holomorphic in $s \in \mathbb{C}$, sum by

$$
\sum_{j=0}^{\infty}\left[c_{U^{-j} \sigma_{1}}(\lambda)^{-s}+\cdots+c_{U^{-j} \sigma_{2^{d}-1}}(\lambda)^{-s}\right] .
$$


With $\zeta^{R}(s)$ denoting the Riemann zeta function, we may using Theorem 2 compare

$$
|\sum_{j=0}^{\infty} c_{U^{-j} \sigma_{1}}(\lambda)^{-s}-\operatorname{vol}(Y, \lambda)^{-\frac{s}{2}} \underbrace{\sum_{j=0}^{\infty} j^{-\frac{s}{2}}}_{=\zeta^{R}\left(\frac{s}{2}\right)}|=O\left(\sum_{j=0}^{\infty} \frac{1}{j^{3 s / 5}}\right)
$$

whence the difference is holomorphic for $\operatorname{Re}(s)>\frac{5}{3}$. The corollary now follows on knowing $s=2$ to be the only pole of the Riemann zeta function $\zeta^{R}\left(\frac{s}{2}\right)$ with residue 1.

\subsection{The ellipsoid example}

We close by presenting an example with $O(1)$ asymptotics, and where the corresponding $\zeta_{E C H}$ function extends meromorphically to all of $\mathbb{C}$.

Consider the symplectic ellipsoid

$$
E(a, b):=\left\{\frac{\left|z_{1}\right|^{2}}{a}+\frac{\left|z_{2}\right|^{2}}{b} \leq 1\right\} \subset \mathbb{C}^{2}=\mathbb{R}^{4} .
$$

The symplectic form on $\mathbb{R}^{4}$ has a standard primitive

$$
\lambda_{s t d}=\frac{1}{2} \sum_{i=1}^{2}\left(x_{i} d y_{i}-y_{i} d x_{i}\right) .
$$

This restricts to $\partial E(a, b)$ as a contact form, and the ECH spectrum of ( $\partial E(a, b), \lambda)$ is known. Specifically, let $N(a, b)$ be the sequence whose $j^{\text {th }}$ element (indexed starting at $j=0)$ is the $(j+1)^{s t}$ smallest element in the matrix

$$
(m a+n b)_{(m, n) \in \mathbb{Z}_{\geq 0} \times \mathbb{Z}_{\geq 0} .}
$$

Then, the ECH spectrum $S_{\partial E(a, b)}$ is precisely the values in $N(a, b)$. Moreover, the homology

$$
E C H_{*}(\partial E(a, b))
$$

has a canonical $\mathbb{Z}$-grading, such that the empty set of Reeb orbits has grading 0 , and it is known to have one generator $\sigma_{j}$ in each grading $2 j$, see [22]. The spectral invariant associated to $\sigma_{j}$ is precisely the $j^{\text {th }}$ element in the sequence $N(a, b)$.

With this understood, we now have the following proposition (cf. [12, Remark 3.2.5.iii]). 
Proposition 15 For any sequence $\left\{\sigma_{j}\right\}_{j=1}^{\infty}$ of nonzero classes in $E C H(\partial E(a, b))$ with gradings $\operatorname{gr}^{\mathbb{Q}}\left(\sigma_{j}\right)=2 j$ one has $e(j)=O(1)$. Furthermore, for a/b irrational one has

$$
\lim _{j \rightarrow \infty} e(j)=\frac{a+b}{2}
$$

Proof This was essentially proved in the reference above, but we reproduce the proof here for completeness. Assume that $a / b$ is irrational. If $t=c_{\sigma_{j}}$, then by the above description, the grading of $\sigma_{j}$ is precisely twice the number of terms in $N(a, b)$ that have value less than $t$. With this understood, the example follows from [18, Lemma 2.1].

When $a / b$ is rational, a similar argument still works to show $O(1)$ asymptotics. Namely, if $t=c_{\sigma_{j}}$, then by above, the grading of $\sigma_{j}$ is precisely twice the number of terms in $N(a, b)$ that have value less than $t$, up to an error no larger than some constant multiple of $\sqrt{j}$. Now apply [6, Theorem 2.10].

Proposition 16 The ECH $\zeta$ function $\zeta_{E C H}$ for $E C H(\partial E(a, b))$ has a meromorphic continuation to all of $\mathbb{C}$. It has exactly two poles, at $s=1$ and $s=2$, with residues

$$
\operatorname{Res}_{s=2} \zeta_{E C H}(s ; Y, \lambda, \Gamma)=\frac{1}{a b}, \quad \operatorname{Res}_{s=1} \zeta_{E C H}(s ; Y, \lambda, \Gamma)=\frac{1}{2}\left(\frac{1}{a}+\frac{1}{b}\right) .
$$

Proof The ECH zeta function in this example

$$
\begin{aligned}
\zeta_{E C H}(s ; Y, \lambda, \Gamma) & =\sum_{m, n \in \mathbb{N}}(m a+n b)^{-s} \\
& =\frac{1}{2}\left[\zeta^{B}(s, a \mid a, b)+\zeta^{B}(s, b \mid a, b)-a^{-s} \zeta^{R}(s)-b^{-s} \zeta^{R}(s)\right]
\end{aligned}
$$

is given in terms of the classical zeta functions of Riemann and Barnes [5,33]

$$
\zeta^{B}(s, w \mid a, b):=\sum_{m, n \in \mathbb{N}_{0}}(w+m a+n b)^{-s}, w \in \mathbb{R}_{>0}
$$

Thus $\zeta_{E C H}(s ; Y, \lambda, \Gamma)(4.15)$ is known to possess a meromorphic continuation to the entire complex plane in this example. Its only two poles are at $s=1,2$ with residues

$$
\begin{aligned}
\operatorname{Res}_{S=2} \zeta_{E C H}(s ; Y, \lambda, \Gamma) & =\frac{1}{a b} \\
\operatorname{Res}_{S=1} \zeta_{E C H}(s ; Y, \lambda, \Gamma) & =\frac{1}{2}\left(\frac{1}{a}+\frac{1}{b}\right)
\end{aligned}
$$


respectively; while its values at the non-positive integers are also known [39, Corollary 2.4]. In particular its value at zero is

$$
\zeta_{E C H}(0 ; Y, \lambda, \Gamma)=\frac{1}{4}+\frac{1}{12}\left(\frac{b}{a}+\frac{a}{b}\right)
$$

\subsection{Sub-leading asymptotics in symplectic embedding problems}

A symplectic embedding of one symplectic manifold $\left(X_{1}, \omega_{1}\right)$ into another $\left(X_{2}, \omega_{2}\right)$ is a smooth embedding

$$
\Psi: X_{1} \rightarrow X_{2}
$$

such that $\Psi^{*} \omega_{2}=\omega_{1}$. When the $\left(X_{i}, \omega_{i}\right)$ are symplectic 4-manifolds, ECH can be used to assign a sequence of nonnegative real numbers

$$
0=c_{0}\left(X_{i}, \omega_{i}\right) \leq c_{1}\left(X_{i}, \omega_{i}\right) \leq \cdots \leq \infty
$$

called ECH capacities. The ECH capacities are monotone under symplectic embeddings, and so are embedding obstructions. Moreover, for a large class of symplectic four-manifolds, for example symplectic ellipsoids, the obstruction they give is sharp [11].

We first explain a direct consequence of Theorems 1 and 2 for ECH capacities. Define a Liouville domain to be a compact symplectic 4-manifold $(X, \omega)$ with oriented boundary, such that $\omega$ is exact, and $\left.\omega\right|_{\partial X}=d \lambda$ for a contact form $\lambda$.

Corollary 17 Let $(X, \omega)$ be a Liouville domain with all ECH capacities finite. Then

$$
c_{k}(X, \omega)=2 \sqrt{k \operatorname{vol}(X, \omega)}+O\left(k^{2 / 5}\right) .
$$

as $k \rightarrow \infty$.

Proof The corollary is a straightforward application of Theorems 1 and 2, given the definitions. Let $\lambda$ be a contact form such that $\left.\omega\right|_{\partial X}=d \lambda$. As explained in [16, Sects. 1.1 and 1.2], there is a degree -2 map $U$ on $E C H(\partial X, \lambda, 0)$, and $c_{k}(X, \omega)$ is by definition the minimum action required to find a nonzero class $\sigma$ with $U^{k} \sigma=[\emptyset]$, where $[\emptyset]$ is the class of the empty set. The hypothesis that all ECH capacities are finite implies that $[\emptyset] \neq 0$.

In the ellipsoid case, where the sub-leading term of the asymptotics can be computed directly, the answer seems to be connected to known symplectic embedding phenomenon.

Example 18 A famous result of McDuff-Schlenk computes the function

$$
c(a):=\min \left\{\lambda \mid E(1, a) \rightarrow B^{4}(\lambda)\right\} .
$$


(Here the arrow refers to a symplectic embedding.) For $a \geq 1$, this determines when precisely a four-dimensional ellipsoid can be symplectically embedded into a fourdimensional ball. They find that the function $c(a)$ begins with an "infinite staircase" determined by the odd-index Fibonacci numbers, accumulating at $a=\tau^{4}$, see [30, Theorem 1.1]. This is a very curious phenomenon; however, the number $\tau^{4}$ has a natural explanation in terms of the role of sub-leading asymptotics in this problem.

To elaborate, by Theorem 1, see (4.16), the coefficient of the leading asymptotics for the $c_{k}$ for $E(1, a)$ is $\sqrt{2 a}$; and by Proposition 15 , the coefficient of the sub-leading asymptotics is $\frac{1+a}{2}$. If we take $\lambda=\sqrt{a}$, then the leading asymptotics for the $c_{k}$ for $B^{4}(\lambda, \lambda)$ is also given by $2 \sqrt{a}$, and the sub-leading asymptotics should be regarded as given by $\frac{3}{2} \lambda$. Thus, the leading and sub-leading asymptotics agree precisely at the solutions of the equation

$$
a+1=3 \sqrt{a}
$$

the only solution of this equation with $a>1$ is $a=\tau^{4}$.

A similar analysis holds for the infinite staircases in [17], and so one could hope to study the sub-leading term to find similar phenomena for other embedding problems, which is a subject of ongoing work [13].

Example 19 In dimension greater than 4, little is known even about the question of when one symplectic ellipsoid embeds into another. The first author has been studying this in joint work for ellipsoids where most of the arguments are infinite. As explained in [12, Remark 1.1.2.(iii), 3.2.5.(iii)], the obstruction coming from the sub-leading term seems to be related to what embeddings exist in this context.

Example 20 Another curious phenomenon in the McDuff-Schlenk computation mentioned above is that

$$
c(a)=\sqrt{a}, \quad a \geq(17 / 6)^{2} .
$$

In other words, if an ellipsoid is sufficiently stretched, then all symplectic embedding obstructions vanish for this embedding problem into a ball except for the classical volume obstruction. This is natural to expect, in view of our computation of the subleading asymptotics: as the ellipsoid becomes more and more stretched, the sub-leading asymptotics of the $c_{k}$ get smaller relative to the sub-leading asymptotics of a ball of the same volume, and so one does not expect further obstructions.

This observation has potential applications for proving flexibility results for embeddings into other targets. For example, define a convex toric domain to be a region

$$
X_{\Omega}:=\left\{\left(z_{1}, z_{2}\right) \in \mathbb{C}^{2} \mid\left(\pi\left|z_{1}\right|^{2}, \pi\left|z_{2}\right|^{2}\right) \in \Omega\right\},
$$

where $\Omega$ is a convex region in the first quadrant of $\mathbb{R}^{2}$ containing the origin in its interior. The first author has conjectured that for any convex toric domain, the only obstruction to symplectically embedding a sufficiently stretched ellipsoid is the volume, and work in progress aims to prove this conjecture and related conjectures, for 
example to stretched concave domains, by studying the sub-leading asymptotics of ECH capacities, since the obstruction coming from ECH capacities is known to be sharp for this problem [11].

Acknowledgements The first author thanks M. Hutchings, D. McDuff, and W. Sun for very helpful discussion.

Funding Open Access funding enabled and organized by Projekt DEAL.

Open Access This article is licensed under a Creative Commons Attribution 4.0 International License, which permits use, sharing, adaptation, distribution and reproduction in any medium or format, as long as you give appropriate credit to the original author(s) and the source, provide a link to the Creative Commons licence, and indicate if changes were made. The images or other third party material in this article are included in the article's Creative Commons licence, unless indicated otherwise in a credit line to the material. If material is not included in the article's Creative Commons licence and your intended use is not permitted by statutory regulation or exceeds the permitted use, you will need to obtain permission directly from the copyright holder. To view a copy of this licence, visit http://creativecommons.org/licenses/by/4.0/.

\section{Appendix $A$. The $\mathbb{Q}$-grading and the $\eta$ invariant}

In this appendix we give a formula for the absolute grading $\mathrm{gr}^{\mathbb{Q}}$ on monopole Floer groups of torsion spin-c structures (from [26, Sect. 28.3]) in terms of a relevant eta invariant. First to recall the definition of $\mathrm{gr}^{\mathbb{Q}}(\mathfrak{a}), \mathfrak{a}=(A, s, \Phi)$, choose a four manifold $X$ which bounds $Y$ and form the manifold with cylindrical end $Z=X \cup$ $\left(Y \times[0, \infty)_{t}\right)$. Choose a metric $g^{T Z}$ on $Z$ which is of product type $g^{T Z}=g^{T X}+d t^{2}$ on the cylindrical end. Choose a spin-c structure $\left(S^{T Z}, c^{Z}\right)$ over $Z$ which is of the form

$$
\begin{aligned}
S^{T Z} & =S_{+}^{T Z} \oplus S_{-}^{T Z} ; \quad S_{+}^{T Z}=S_{-}^{T Z}=S^{T Y}, \\
c^{Z}(\alpha) & =\left[\begin{array}{cc}
0 & c^{Y}(\alpha) \\
c^{Y}(\alpha) & 0
\end{array}\right], \quad \alpha \in T^{*} Y \\
c^{Z}(d t) & =\left[\begin{array}{cc}
0 & -I \\
I & 0
\end{array}\right]
\end{aligned}
$$

over the cylindrical end and a spin-c connection on $S^{T Z}$ of the form $B=d t \wedge \partial_{t}+A \oplus A$ on the cylindrical end. One may now form the Fredholm elliptic operator

$$
d^{*}+d^{+}+D_{B}^{+}: L_{1}^{2}\left(Z ; \Lambda_{T Z}^{1} \oplus S_{+}^{T Z}\right) \rightarrow L^{2}\left(Z ; \Lambda_{T Z}^{0} \oplus \Lambda_{T Z}^{2,+} \oplus S_{-}^{T Z}\right)
$$

The absolute grading of $\left[\left(A, 0, \Phi_{0}^{A}\right)\right] \in \mathfrak{C}^{s}$, with $A^{t}$ flat and $\Phi_{0}^{A}$ the first positive eigenvector of $D_{A}$, is given in terms of this operator. The precise formula [26, Definition 28.3.1] simplifies to

$$
\operatorname{gr}^{\mathbb{Q}}\left[\left(A, 0, \Phi_{0}^{A}\right)\right]=-2 \operatorname{ind}\left(D_{B}^{+}\right)+\frac{1}{4}\left\langle c_{1}\left(S^{+}\right), c_{1}\left(S^{+}\right)\right\rangle-\frac{1}{4} \sigma(Z)
$$


with $\sigma(Z)$ denoting the signature of $Z, S^{+}$the bundle $S_{+}^{T Z}$ from above, and ind denoting the complex index, namely the difference in complex dimensions, compare [10, Sect. 3.4].

The APS index theorem for spin-c Dirac operators now gives

$$
\operatorname{ind}\left(D_{B}^{+}\right)=\frac{1}{8}\left\langle c_{1}\left(S^{+}\right), c_{1}\left(S^{+}\right)\right\rangle-\frac{1}{24} \int_{X} p_{1}+\frac{\eta\left(D_{A}\right)}{2}
$$

The APS signature theorem for the manifold $X$ with boundary also gives

$$
-\frac{1}{24} \int_{X} p_{1}=-\frac{1}{8} \sigma(Z)-\frac{1}{8} \eta_{Y}
$$

where $\eta_{Y}$ is the eta invariant of the odd signature operator on $C^{\infty}\left(Y ; T^{*} Y \oplus \mathbb{R}\right)$ sending

$$
(a, f) \mapsto\left(* d a-d f,-d^{*} a\right)
$$

Combining the above we have

$$
\operatorname{gr}^{\mathbb{Q}}\left[\left(A, 0, \Phi_{0}^{A}\right)\right]=-\eta\left(D_{A}\right)+\frac{1}{4} \eta_{Y}
$$

A reducible generator $\left[\mathfrak{a}_{k}\right]=\left[\left(A, 0, \Phi_{k}^{A}\right)\right] \in \mathfrak{C}^{s}$ however has $\frac{1}{2} F_{A^{t}}=-d \mu$ and $\Phi_{k}^{A}$ the $k$ th eigenvector of $D_{A}$. Hence,

$$
\operatorname{gr}^{\mathbb{Q}}\left[\left(A, 0, \Phi_{k}^{A}\right)\right]=2 k+\left(-\eta\left(D_{A}\right)+\frac{1}{4} \eta_{Y}\right)-2 \operatorname{sf}\left\{D_{A_{s}}\right\}_{0 \leq s \leq 1}
$$

where $D_{A_{s}}$ is a family of Dirac operators, associated to a family of connections starting at the flat connection and ending at one satisfying $\frac{1}{2} F_{A^{t}}=-d \mu$. Hence, by interpreting this spectral flow as an index through another application of Atiyah-Patodi-Singer [4, p. 95], and applying [3, Eq. 4.3] to compute this index, we get

$$
\operatorname{gr}^{\mathbb{Q}}\left[\left(A, 0, \Phi_{k}^{A}\right)\right]=2 k-\eta\left(D_{A}\right)+\frac{1}{4} \eta_{Y}-\frac{1}{2 \pi^{2}} C S(A)
$$

as the absolute grading of a reducible generator.

The absolute grading of an irreducible generator $\left[\mathfrak{a}^{\prime}\right]=\left(A^{\prime}, s, \Phi^{\prime}\right), s \neq 0$, is then given by

$$
\operatorname{gr}^{\mathbb{Q}}\left[\mathfrak{a}^{\prime}\right]=\operatorname{gr}^{\mathbb{Q}}\left[\mathfrak{a}_{0}\right]-2 \operatorname{sf}\left\{\widehat{\mathcal{H}}_{\left(A_{\varepsilon}, \Psi_{\varepsilon}\right)}\right\}_{0 \leq \varepsilon \leq 1}
$$

in terms of spectral flow of the Hessians (2.4) for a path $\left(A_{\varepsilon}, \Psi_{\varepsilon}\right) \in \mathcal{A}(Y, \mathfrak{s}) \times C^{\infty}(S)$, $\varepsilon \in[0,1]$ starting at $\left[\mathfrak{a}_{0}\right]=\left[\left(A_{0}, 0\right)\right]$ and ending at $\left(A^{\prime}, s \Phi^{\prime}\right)$. As above, we can 
interpret this spectral flow as an index; this time, to compute the relevant index, we need to apply [3, Theorem 3.10], which gives that the above is equal to

$$
\operatorname{gr}^{\mathbb{Q}}\left[\mathfrak{a}^{\prime}\right]=-\eta\left(\widehat{\mathcal{H}}_{\left(A, s \Phi^{\prime}\right)}\right)+\frac{5}{4} \eta_{Y}-2 \int_{Y \times[0,1]_{\varepsilon}} \rho_{0} .
$$

Here $\rho_{0}$ is the usual Atiyah-Singer integrand, namely the local index density defined as the constant term in the small time expansion of the local supertrace $\operatorname{str}\left(e^{-t \mathcal{D}^{2}}\right)$ with

$$
\mathcal{D}=\left[\begin{array}{r}
{ }^{-1} \\
1
\end{array}\right] \partial_{\varepsilon}+\left[\begin{array}{l}
1 \\
1
\end{array}\right] \widehat{\mathcal{H}}_{\left(A_{\varepsilon}, \Psi_{\varepsilon}\right)},
$$

and where $\left(A_{\epsilon}, \Psi_{\epsilon}\right)$ is the chosen path of configurations. To compute the index density we choose a path of the form

$$
\left(A_{\varepsilon}, \Psi_{\varepsilon}\right)= \begin{cases}\left(A+2 \varepsilon\left(A^{\prime}-A\right), 0\right) ; & 0 \leq \varepsilon \leq \frac{1}{2} \\ \left(A^{\prime},(2 \varepsilon-1) \Psi\right) ; & \frac{1}{2} \leq \varepsilon \leq 1\end{cases}
$$

On the interval $\left[0, \frac{1}{2}\right]$, the integral of the local density is given by the usual local index theorem: as above, we have

$$
-2 \int_{Y \times\left[0, \frac{1}{2}\right]_{\varepsilon}} \rho_{0}=-\frac{1}{2 \pi^{2}} C S(A) .
$$

On the other hand, for the calculation on $Y \times\left[\frac{1}{2}, 1\right]_{\varepsilon}$, we have $\rho_{0}=0$. To see this, first note $\mathcal{D}^{2}=-\partial_{\varepsilon}^{2}+\widehat{\mathcal{H}}_{\left(A_{\varepsilon}, \Psi_{\varepsilon}\right)}^{2}+\left[\begin{array}{cc}-1 & \\ & 1\end{array}\right] 2 M_{\Psi}$ gives

$$
\operatorname{str}\left(e^{-t \mathcal{D}^{2}}\right)=\operatorname{tr}\left(e^{-t\left[-\partial_{\varepsilon}^{2}+\widehat{\mathcal{H}}_{\left(A_{\varepsilon}, \Psi_{\varepsilon}\right)}^{2}-2 M_{\Psi}\right]}-e^{-t\left[-\partial_{\varepsilon}^{2}+\widehat{\mathcal{H}}_{\left(A_{\varepsilon}, \Psi_{\varepsilon}\right)}^{2}+2 M_{\Psi}\right]}\right)
$$

Duhamel's principle then gives that the coefficients in the small time heat kernel expansion of the difference above are of the form

$$
\left[\begin{array}{lll}
0 & 0 & * \\
0 & 0 & * \\
* & * & 0
\end{array}\right]
$$

with respect to the decomposition $i T^{*} Y \oplus \mathbb{R} \oplus S$.

Hence we have in summary:

$$
\operatorname{gr}^{\mathbb{Q}}[\mathfrak{a}]= \begin{cases}2 k-\eta\left(D_{A}\right)+\frac{1}{4} \eta_{Y}-\frac{1}{2 \pi^{2}} C S(A) ; & \mathfrak{a}=\left(A, 0, \Phi_{k}^{A}\right) \in \mathfrak{C}^{s}, \\ -\eta\left(\widehat{\mathcal{H}}_{(A, s \Phi)}\right)+\frac{5}{4} \eta_{Y}-\frac{1}{2 \pi^{2}} C S(A) ; & \mathfrak{a}=(A, s, \Phi) \in \mathfrak{C}^{o}, s \neq 0 .\end{cases}
$$




\section{References}

1. Anantharaman, N.: Precise counting results for closed orbits of Anosov flows. Ann. Sci. École Norm. Sup. (4) 33, 33-56 (2000)

2. Asaoka, M., Irie, K.: A $C^{\infty}$ closing lemma for Hamiltonian diffeomorphisms of closed surfaces. Geom. Funct. Anal. 26, 1245-1254 (2016)

3. Atiyah, M.F., Patodi, V.K., Singer, I.M.: Spectral asymmetry and Riemannian geometry. I. Math. Proc. Camb. Philos. Soc. 77, 43-69 (1975)

4. Atiyah, M.F., Patodi, V.K., Singer, I.M.: Spectral asymmetry and Riemannian geometry. III. Math. Proc. Camb. Philos. Soc. 79, 71-99 (1976)

5. Barnes, E.W.: The theory of the double gamma function. Philos. Trans. R. Soc. Lond. Ser. A Contain. Pap. Math. Phys. Character 196, 265-387 (1901)

6. Beck, M., Robins, S.: Computing the continuous discretely, Undergraduate Texts in Mathematics, Springer, New York, 2nd edn. Integer-point enumeration in polyhedra, With illustrations by David Austin (2015)

7. Berline, N., Getzler, E., Vergne, M.: Heat kernels and Dirac operators, Grundlehren Text Editions, Springer, Berlin, 2004. Corrected reprint of the 1992 original

8. Bismut, J.-M., Freed, D.S.: The analysis of elliptic families. II. Dirac operators, eta invariants, and the holonomy theorem. Commun. Math. Phys. 107, 103-163 (1986)

9. Cai, Y.: Prime geodesic theorem. J. Théor. Nombres Bordeaux 14, 59-72 (2002)

10. Cristofaro-Gardiner, D.: The absolute gradings on embedded contact homology and Seiberg-Witten Floer cohomology. Algebr. Geom. Topol. 13, 2239-2260 (2013)

11. Cristofaro-Gardiner, D.: Symplectic embeddings from concave toric domains into convex ones. arXiv:1409.4378 (2014)

12. Cristofaro-Gardiner, D., Hind, R., McDuff, D.: The ghost stairs stabilize to sharp symplectic embedding obstructions. J. Topol. 11, 309-378 (2018)

13. Cristofaro-Gardiner, D., Holm, T., Mandini, A., Pires, A.R.: Symplectic embeddings and infinite staircases (in preparation)

14. Cristofaro-Gardiner, D., Hutchings, M.: From one Reeb orbit to two. J. Differ. Geom. 102, 25-36 (2016)

15. Cristofaro-Gardiner, D., Hutchings, M., Pomerleano, D.: Torsion contact forms in three dimensions have two or infinitely many Reeb orbits. Geom. Topol. 23, 3601-3645 (2019)

16. Cristofaro-Gardiner, D., Hutchings, M., Ramos, V.G.B.: The asymptotics of ECH capacities. Invent. Math. 199, 187-214 (2015)

17. Cristofaro-Gardiner, D., Kleinman, A.: Ehrhart polynomials and symplectic embeddings of ellipsoids. arXiv:1307.5493 (2013)

18. Cristofaro-Gardiner, D., Li, T., Stanley, R.: Irrational triangles with polynomial Ehrhart functions (2018)

19. Dyatlov, S., Zworski, M.: Dynamical zeta functions for Anosov flows via microlocal analysis. Ann. Sci. Éc. Norm. Supér. (4) 49, 543-577 (2016)

20. Giulietti, P., Liverani, C., Pollicott, M.: Anosov flows and dynamical zeta functions. Ann. Math. (2) 178, 687-773 (2013)

21. Hutchings, M.: Quantitative embedded contact homology. J. Differ. Geom. 88, 231-266 (2011)

22. Hutchings, M.: Lecture notes on embedded contact homology. Contact and symplectic topology, vol. 26 of Bolyai Soc. Math. Stud., János Bolyai Math. Soc., Budapest, pp. 389-484 (2014)

23. Irie, K.: Dense existence of periodic Reeb orbits and ECH spectral invariants. J. Mod. Dyn. 9, 357-363 (2015)

24. Iwaniec, H.: Prime geodesic theorem. J. Reine Angew. Math. 349, 136-159 (1984)

25. Katsuda, A., Sunada, T.: Closed orbits in homology classes. Inst. Hautes Études Sci. Publ. Math., pp. 5-32 (1990)

26. Kronheimer, P., Mrowka, T.: Monopoles and Three-Manifolds. New Mathematical Monographs, vol. 10. Cambridge University Press, Cambridge (2007)

27. Lalley, S.P.: Closed geodesics in homology classes on surfaces of variable negative curvature. Duke Math. J. 58, 795-821 (1989)

28. Luo, W.Z., Sarnak, P.: Quantum ergodicity of eigenfunctions on $P S L \_2(\mathbb{Z}) \backslash \mathbb{H}$, Inst. Hautes Études Sci. Publ. Math. (1995), pp. 207-237 
29. Margulis, G.A.: Certain applications of ergodic theory to the investigation of manifolds of negative curvature. Funkcional. Anal. i Priloen. 3, 89-90 (1969)

30. McDuff, D., Schlenk, F.: The embedding capacity of 4-dimensional symplectic ellipsoids. Ann. Math. (2) 175, 1191-1282 (2012)

31. Phillips, R., Sarnak, P.: Geodesics in homology classes. Duke Math. J. 55, 287-297 (1987)

32. Pollicott, M., Sharp, R.: Asymptotic expansions for closed orbits in homology classes. Geom. Dedic. 87, 123-160 (2001)

33. Ruijsenaars, S.N.M.: On Barnes' multiple zeta and gamma functions. Adv. Math. 156, 107-132 (2000)

34. Savale, N.: Spectral Asymptotics for Coupled Dirac Operators. ProQuest LLC, Ann Arbor, MI, Thesis (Ph.D.) - Massachusetts Institute of Technology (2012)

35. Savale, N.: Asymptotics of the Eta invariant. Commun. Math. Phys. 332, 847-884 (2014)

36. Savale, N.: Koszul complexes, Birkhoff normal form and the magnetic Dirac operator. Anal. PDE 10, 1793-1844 (2017)

37. Savale, N.: A Gutzwiller type trace formula for the magnetic Dirac operator. Geom. Funct. Anal. 28, 1420-1486 (2018)

38. Soundararajan, K., Young, M.P.: The prime geodesic theorem. J. Reine Angew. Math. 676, 105-120 (2013)

39. Spreafico, M.: On the Barnes double zeta and Gamma functions. J. Number Theory 129, 2035-2063 (2009)

40. Taubes, C.H.: The Seiberg-Witten equations and the Weinstein conjecture. Geom. Topol. 11, 21172202 (2007)

41. Taubes, C.H.: Embedded contact homology and Seiberg-Witten Floer cohomology I. Geom. Topol. 14, 2497-2581 (2010)

42. Tsai, C.-J.: Asymptotic spectral flow for Dirac operations of disjoint Dehn twists. Asian J. Math. 18, 633-685 (2014)

43. Weifeng, S.: An estimate on energy of min-max Seiberg-Witten Floer generators (Jan. 2018) arXiv: 1801.02301

Publisher's Note Springer Nature remains neutral with regard to jurisdictional claims in published maps and institutional affiliations. 